\title{
Article \\ Combined Application of Rice Husk Biochar and Lime Increases Phosphorus Availability and Maize Yield in an Acidic Soil
}

\author{
Mehnaz Mosharrof ${ }^{1,2}$, Md. Kamal Uddin ${ }^{1, *(\mathbb{D})}$, Muhammad Firdaus Sulaiman ${ }^{1}\left(\mathbb{D}\right.$, Shamim Mia ${ }^{3} \mathbb{D}$, \\ Shordar M. Shamsuzzaman ${ }^{4}$ and Ahmad Numery Ashfaqul Haque ${ }^{1}$ (D)
}

1 Department of Land Management, Faculty of Agriculture, Universiti Putra Malaysia, Serdang SGR 43400, Malaysia; mmd.mehnaz@gmail.com (M.M.); muhdfirdaus@upm.edu.my (M.F.S.); numerybau@gmail.com (A.N.A.H.)

2 Central Laboratory, Soil Resource Development Institute (SRDI), Krishi Khamar Sharak, Dhaka 1215, Bangladesh

3 Department of Agronomy, Patuakhali Science and Technology University, Patuakhali 8602, Bangladesh; smia_agr@pstu.ac.bd

4 Divisional Laboratory, Soil Resource Development Institute (SRDI), Krishi Khamar Sharak, Dhaka 1215, Bangladesh; shamsuzzamansm@gmail.com

* Correspondence: mkuddin07@gmail.com; Tel.: +60-014-929-4350

check for updates

Citation: Mosharrof, M.; Uddin, M.K.; Sulaiman, M.F.; Mia, S.; Shamsuzzaman, S.M.; Haque, A.N.A. Combined Application of Rice Husk Biochar and Lime Increases Phosphorus Availability and Maize Yield in an Acidic Soil. Agriculture 2021, 11, 793. https://doi.org/ 10.3390 /agriculture11080793

Academic Editor: Nguyen V. Hue

Received: 21 June 2021

Accepted: 16 August 2021

Published: 19 August 2021

Publisher's Note: MDPI stays neutral with regard to jurisdictional claims in published maps and institutional affiliations.

Copyright: (c) 2021 by the authors Licensee MDPI, Basel, Switzerland. This article is an open access article distributed under the terms and conditions of the Creative Commons Attribution (CC BY) license (https:// creativecommons.org/licenses/by/ $4.0 /)$.

\begin{abstract}
Biochar, a pyrogenic carbon, has been receiving incremental attention for potential contribution to soil health, agricultural productivity enhancement while mitigating climate change by sequestering carbon and reducing greenhouse gas (GHG) emissions. However, it is not well-known to us how far rice husk biochar (RHB) application rates could increase phosphorus (P) bioavailability and plant performance when co-applied with $\mathrm{P}$ and lime. Here, we present data of a pot experiment consisting of eleven treatments to evaluate RHB, lime, and phosphorus effect on soil phosphorus availability, $\mathrm{CO}_{2}$ emission, nutrient uptake, and yield performance of maize. Co-application of RHB (10 and $\left.15 \mathrm{tha}^{-1}\right)$ and lime (100\% and $75 \%$ ) was made with different rates of $\mathrm{P}(100 \%, 75 \%$, and $50 \%)$. Our result revealed that, at harvest, the combined application of RHB, lime, and phosphorus fertilizer significantly increased soil $\mathrm{pH}, \mathrm{P}$ availability and decreased $\mathrm{Al}$ and Fe toxicity relative to the control while increasing maize yield. The maximum soil $\mathrm{pH}$ increased by $36.75 \%$, the highest available $\mathrm{P}$ increased by $158.75 \%$, whilst, the exchangeable $\mathrm{Al}$ content reduced by $96.84 \%$ compared to the control treatment. However, the difference in biomass production and yield among different lime, RHB, and $P$ were minimal, with the largest grain yield $\left(15.50 \mathrm{tha}^{-1}\right)$ was recorded in the $\mathrm{T}_{6}$ treatments $(75 \%$ lime $+10 \mathrm{tha}^{-1} \mathrm{RHB}+100 \%$ Triple superphosphate). The increment in biomass and grain yield could have occurred due to lime and RHB mediated changes in soil properties, including enhancement of soil $\mathrm{pH}$, availability of $\mathrm{P}$, and other nutrients. This increased availability then increased nutrient uptake and biomass production. Our results suggest that the combined application of lime and RHB could bring favorable changes in soil properties while sacrificing some carbon from soils.
\end{abstract}

Keywords: maize; acid soil; rice husk biochar; lime; Triple superphosphate (TSP); phosphorus availability; yield

\section{Introduction}

Maize is considered a universal crop for its multipurpose uses, including food for human beings and feed for animals [1]. Therefore, it can grow globally in different weather conditions and various soil types [2]. However, soil acidity is one of the main constraints for maize cultivation. Tropical soils are generally influenced by high precipitation and temperature, leading to the loss of basic cations. Therefore, these soils are highly weathered acidic soils [3]. As a result, in this tropical and subtropical region, soil acidification is 
one of the major limiting factors for crop production since soil acidity reduces nutrient availability [4-6].

Several management practices are suggested to ameliorate acidic soils, while liming is considered the easiest soil amendment [7]. Lime is traditionally incorporated into the surface and sub-surface zone of soil to buffer soil pH [8]. However, the effects of lime in the soil are not sustainable since reacidification of soil has been reported due to exhaustion of the liming effects [9]. Therefore, alternative soil amendments, including organic matter addition and recently biochar, have been suggested.

Biochar is a pyrogenic carbon-rich material. It has a large surface area while it carries functional groups. Moreover, in its ash, it contains different mineral nutrients, including basic cations (Ca and $\mathrm{Mg}$ ). Therefore, biochar can potentially buffer soil $\mathrm{pH}$ by adding basic cations and consuming protons with negatively charged functionals groups (e.g., carboxylic and phenolic groups) [10]. In addition, biochar can also change nutrient dynamics that can change the acidification of soil. For instance, biochar can retain ammonium which plants can take. Mineralization and uptake of ammonium $\left(\mathrm{NH}_{4}{ }^{+}\right)$do not cause acidification since mineralization-mediated hydroxyl ions $\left(\mathrm{OH}^{-}\right)$counterbalance the proton released by the plant during $\mathrm{NH}_{4}{ }^{+}$uptake. However, the acidification is doubled if the $\mathrm{NH}_{4}{ }^{+}$ is converted to $\mathrm{NO}_{3}{ }^{-}$and then leached [11]. Therefore, nitrate leaching from soil can be one of the major causes of soil acidification. The role of biochar in alleviating soil acidification could therefore depend on biochar properties, while it has been shown that biochar, after becoming aged in the soils, has a greater potentiality to buffer soil $\mathrm{pH}$. Since biochar's residence time in the soil long ranging from decades to centuries, multiple benefits, including alleviating soil acidity, decreasing the toxic accumulation of $\mathrm{Al}$ and Fe concentration, crop yield improvement, and thus, environmental sustainability can be harvested for a long time [12,13]. These findings underscore the potential benefits of biochar as a soil amendment for carbon sequestration as well as having better soils. One of the most popular examples of such benefits is Terra preta soil in Brazil.

Biochar is an alternate option for alleviating soil acidity and environmental sustainability [12]. It is a carbon-rich, recalcitrant organic material obtained from the thermal degradation of biomass in different temperatures and feedstocks [14,15]. Furthermore, it is an ecologically sound soil amendment due to its quality of resistance to decompose. These attributes potentially contribute to carbon sequestration into the soil for a long [16,17]. It also helps increase soil $\mathrm{pH}$, availability of nutrients, diversity of micro-organisms, and decrease the toxic accumulation of $\mathrm{Al}$ and $\mathrm{Fe}$ concentration [13,18-21].

Phosphorus $(\mathrm{P})$ is an essential macronutrient for the plant, playing a vital role in many physiological processes, including photosynthesis and energy transfer [22]. However, in acid soils, the bioavailability of $\mathrm{P}$ is low due to the fixation of $\mathrm{P}$ with $\mathrm{Al}$ and Fe-bearing minerals [23]. There are several possible ways to increase $\mathrm{P}$ bioavailability in acid soils. These are: (a) an increase of soil $\mathrm{pH}$, (b) intensification of competitive interactions between fixed phosphate and other negatively charged organic molecules (e.g., humic or fulvic acids), and (c) microbial cycling (e.g., mycorrhizal P association). Therefore, the application of organic matter has been shown to increase P bioavailability. Biochar, being an organic soil amendment, could increase $\mathrm{P}$ bioavailability in acid soils by changing soil $\mathrm{pH}$ (as discussed above) and intensification of competitive interactions [10,24]. However, freshly prepared biochar may be less efficient in competing with fixed phosphate since biochar is large with low negative surfaces $[25,26]$.

Soil organic matter content of many highly weathered soils is low. Therefore, the application of pyrogenic carbon as biochar could increase the organic matter status of the soil. However, the application of biochar could alter soil carbon dynamics since biochar changes substrate pools and nutrient dynamics. For instance, an increase in soil $\mathrm{pH}$ can increase $\mathrm{CO}_{2}$ gas emission considering the organic matter and soil microbes decomposition [27], while positive, negative, and minimal responses of $\mathrm{CO}_{2}$ emission from biochar amended soil with attribution of several potential mechanisms [28-30]. Therefore, 
a clear understanding of biochar-mediated impacts on carbon mineralization from acidic soil is needed for large-scale field application.

To meet the increased food demand globally, approximately 15 million tons of $\mathrm{P}$ chemical fertilizer provide every year to farmland [31]. However, due to lack of proper knowledge regarding the appropriate rate of $P$ fertilizer, repeated and excess $P$ fertilizer addition to cultivable land causes loss of $P$ through different pathways, including leaching and runoff. As a result, water pollution occurs due to increased P concentration in the water [32]. Furthermore, phosphate fertilizers are usually produced from rock phosphate, which is projected to exhaust in the near future. Therefore, rational use of phosphate fertilizer is needed. Considering these facts, a pot trial was conducted with acidic soil having the objectives to determine the effect of RHB, lime, and phosphorus on soil phosphorus availability, nutrient uptake, and yield performance of maize and to examine the emission of soil $\mathrm{CO}_{2}$ gas.

\section{Materials and Methods}

\subsection{Experimental Site}

The pot trial was conducted in the new glasshouse, Faculty of Agriculture, Universiti Putra Malaysia, Serdang, Selangor. The experimental site was located at $2^{\circ} 98^{\prime} 36.6^{\prime \prime} \mathrm{N}$ (north) latitude and $101^{\circ} 73^{\prime} 81.9^{\prime \prime} \mathrm{E}$ (east) longitudes with an elevation of $56.8 \mathrm{~m}$ from sea level at the west coast of Peninsular Malaysia. The local climate is hot, humid tropic, and the average minimum temperature was around $22{ }^{\circ} \mathrm{C}$, and the average maximum temperature was $36^{\circ} \mathrm{C}$, relative humidity was $78.87 \%$ during the whole experiment.

\subsection{Soil Collection and Preparation}

The Bungor (Typic Paleudult; Order: Ultisol) soil series was collected in depth from 0-20 cm from Taman Partanian, Universiti Putra Malaysia, Puchong, Selangor $\left(2^{\circ} 58^{\prime} 59.7^{\prime \prime} \mathrm{N}\right.$ latitude; $101^{\circ} 38^{\prime} 47.5^{\prime \prime}$ E longitude). The soil sample was air-dried, pulverized, and sieved to $<2 \mathrm{~mm}$ before chemical characterization and prior to treatment. Thirty-two plastic containers (Height $38 \mathrm{~cm}$, width $30 \mathrm{~cm}$, and diameter $32 \mathrm{~cm}$ ) were used with $20 \mathrm{~kg}$ of soil. Each container was drilled with three holes at the bottom to permit leachate to flow out. The moisture content was maintained using a portable moisture meter (FieldScout TDR 150 Soil Moisture Meter). Biochar and lime were incorporated with soil before two weeks of maize seeds were sowing. Four seeds were planted in $2 \mathrm{~cm}$ depth of soil surface in each pot and thinned to one plant per pot after 7 days of emergence based on the healthy appearance. N-P-K fertilizer was applied in each pot recommended by Pedram [33], as follows: urea $\left(140 \mathrm{~kg} \mathrm{ha}^{-1} \mathrm{~N}\right)$, triple superphosphate $\left(100 \mathrm{~kg} \mathrm{ha}^{-1} \mathrm{P}_{2} \mathrm{O}_{5}\right)$, and muriate of potash $\left(120 \mathrm{~kg} \mathrm{ha}^{-1} \mathrm{~K}_{2} \mathrm{O}\right)$. The full dose of $\mathrm{P}$ and $\mathrm{K}$ fertilizer were applied as a basal dose one day before the seeds were sown. $\mathrm{N}$ fertilizer was applied in two equal splits on the 10th and 28th days after sowing (DAS). Based on the lime requirement test, dolomitic limestone was applied at $66 \mathrm{~g}(100 \%)$ and $49.5 \mathrm{~g}(75 \%)$ [34]. Weeding was carried out manually from each pot immediately after the emergence of weeds. The experiment was conducted from August 2020 to November 2020. The physico-chemical properties of the initial soil are shown in Table 1. 
Table 1. Selected physicochemical properties of initial soil.

\begin{tabular}{|c|c|}
\hline Properties & Soil \\
\hline Textural Class & Sandy clay loam \\
\hline$\%$ Sand & 69.27 \\
\hline$\%$ Silt & 2.28 \\
\hline \% Clay & 28.44 \\
\hline $\mathrm{pH}$ & 4.61 \\
\hline $\operatorname{CEC~}\left(\mathrm{cmol}_{\mathrm{C}} \mathrm{kg}^{-1}\right)$ & 5.77 \\
\hline Total C (\%) & 1.41 \\
\hline Total N (\%) & 0.07 \\
\hline Total S (\%) & 0.05 \\
\hline Exchangeable $\mathrm{K}\left(\mathrm{cmol}_{\mathrm{c}} \mathrm{kg}^{-1}\right)$ & 0.22 \\
\hline Exchangeable $\mathrm{Ca}\left(\mathrm{cmol}_{\mathrm{C}} \mathrm{kg}^{-1}\right)$ & 1.46 \\
\hline Exchangeable $\mathrm{Mg}\left(\mathrm{cmol}_{\mathrm{c}} \mathrm{kg}^{-1}\right)$ & 0.42 \\
\hline Exchangeable $\mathrm{Al}\left(\mathrm{cmol}_{\mathrm{C}} \mathrm{kg}^{-1}\right)$ & 2.49 \\
\hline Available $\mathrm{P}\left(\mathrm{mg} \mathrm{kg}^{-1}\right)$ & 5.21 \\
\hline Extractable Fe $\left(\mathrm{mg} \mathrm{kg}^{-1}\right)$ & 99.44 \\
\hline Extractable Mn (mg kg $\left.{ }^{-1}\right)$ & 4.64 \\
\hline $\mathrm{NH}_{4}{ }^{+}-\mathrm{N}\left(\mathrm{mg} \mathrm{kg}^{-1}\right)$ & 16.41 \\
\hline $\mathrm{NO}_{3}^{-}-\mathrm{N}\left(\mathrm{mg} \mathrm{kg}^{-1}\right)$ & 11.37 \\
\hline
\end{tabular}

\subsection{Rice Husk Biochar Collection and Characterization}

Rice husk biochar (RHB) was used in this pot experiment, made from locally available feedstock in Malaysia. This biochar was obtained from Sendi Enterprise (Sungai Burong, Selangor, Malaysia) via a pyrolysis process at $300{ }^{\circ} \mathrm{C}$. The physicochemical properties of RHB are shown below (Table 2).

Table 2. Selected physical and chemical properties of RHB.

\begin{tabular}{cc}
\hline Properties & RHB \\
\hline Moisture Content $(\%)$ & 6 \\
Ash Content $(\%)$ & 32.40 \\
pH & 8.15 \\
CEC $\left(\mathrm{cmol}_{\mathrm{c}} \mathrm{kg}^{-1}\right)$ & 48.12 \\
Total C $(\%)$ & 24.86 \\
Total N $(\%)$ & 1.13 \\
Total S $(\%)$ & 0.15 \\
Exchangeable K $\left(\mathrm{cmol}_{\mathrm{c}} \mathrm{kg}^{-1}\right)$ & 17.45 \\
Exchangeable Ca $\left(\mathrm{cmol}_{\mathrm{c}} \mathrm{kg}^{-1}\right)$ & 19.46 \\
Exchangeable Mg $\left(\mathrm{cmol}_{\mathrm{c}} \mathrm{kg}^{-1}\right)$ & 13.96 \\
Available P $\left(\mathrm{mg} \mathrm{kg}^{-1}\right)$ & 3098.40 \\
Extractable Fe $\left(\mathrm{mg} \mathrm{kg}^{-1}\right)$ & 43.06 \\
Extractable Mn $\left(\mathrm{mg} \mathrm{kg}^{-1}\right)$ & 23.51 \\
\hline
\end{tabular}

\subsection{Experimental Layout and Treatment}

A single factor experiment was arranged in a randomized complete block design (RCBD) with four replications. The detailed treatments are presenting in Table 3. 
Table 3. Teatments applied in the pot experiment.

\begin{tabular}{|c|c|}
\hline Treatment Code & Description of the Treatments \\
\hline $\mathrm{T}_{1}$ & Control (no treatments and fertilizer) \\
\hline $\mathrm{T}_{2}$ & Recommended rate of NPK $\left(\mathrm{tha}^{-1}\right)$ \\
\hline $\mathrm{T}_{3}$ & $100 \%$ dolomitic limestone $+10 \mathrm{t} \mathrm{ha}^{-1}$ rice husk biochar $+100 \%$ TSP \\
\hline $\mathrm{T}_{4}$ & $100 \%$ dolomitic limestone $+10 \mathrm{tha}^{-1}$ rice husk biochar $+75 \%$ TSP \\
\hline $\mathrm{T}_{5}$ & $100 \%$ dolomitic limestone $+10 \mathrm{tha}^{-1}$ rice husk biochar $+50 \%$ TSP \\
\hline $\mathrm{T}_{6}$ & $75 \%$ dolomitic limestone $+10 \mathrm{tha}^{-1}$ rice husk biochar $+100 \%$ TSP \\
\hline $\mathrm{T}_{7}$ & $75 \%$ dolomitic limestone $+10 \mathrm{tha}^{-1}$ rice husk biochar $+75 \%$ TSP \\
\hline $\mathrm{T}_{8}$ & $75 \%$ dolomitic limestone $+10 \mathrm{tha}^{-1}$ rice husk biochar $+50 \%$ TSP \\
\hline $\mathrm{T}_{9}$ & $75 \%$ dolomitic limestone $+15 \mathrm{tha}^{-1}$ rice husk biochar $+100 \%$ TSP \\
\hline $\mathrm{T}_{10}$ & $75 \%$ dolomitic limestone $+15 \mathrm{tha}^{-1}$ rice husk biochar $+75 \%$ TSP \\
\hline $\mathrm{T}_{11}$ & $75 \%$ dolomitic limestone $+15 \mathrm{t} \mathrm{ha}^{-1}$ rice husk biochar $+50 \%$ TSP \\
\hline
\end{tabular}

\subsection{Soil Analysis}

The hydrometer method was used to determine the soil particle-size distribution [35]. Then, the textural classification was based on the United States Department of Agriculture (USDA) soil texture triangle of size classes as clay $(<2 \mu \mathrm{m})$, silt $(2-50 \mu \mathrm{m})$, and sand as $(50-2000 \mu \mathrm{m})$ [36]. Finally, the bulk density of soil was determined by using a core-ring method [37].

Soil $\mathrm{pH}$ was measured in a 1:2.5 (weight/volume basis) soil: distilled water ratio using a glass electrode $\mathrm{pH}$ meter (Model: Eijkelkamp Multimeter 18.52.01) [38]. Soil CEC was determined using the ammonium acetate leaching method at $\mathrm{pH} 7$. The leachate was analyzed for $\mathrm{NH}_{4}{ }^{+}$concentration using the Auto-analyzer (AA) to determine soil CEC. Soil total carbon (TC), total nitrogen (TN), and total sulphur (TS) were measured by dry combustion method (Dumas method) using a LECO TrueSpec CNS auto analyzer (LECO Corporation, St. Joseph, MI, USA) using air dry and ground soil. The exchangeable cation $\mathrm{Ca}, \mathrm{Mg}$, and $\mathrm{K}$ was extracted using a 5:50 ratio of soil: ammonium acetate $\left(\mathrm{NH}_{4} \mathrm{OAc}\right)$ buffered solution at $\mathrm{pH} 7$, in which the basic cations adsorbed in soil was replaced by $\mathrm{NH}_{4}{ }^{+}$ ion [39], and concentration was determined by inductively coupled plasma optical emission spectroscopy (ICP-OES, PerkinElmer, Inc., Waltham, MA, USA). Soil exchangeable Al was extracted using $1 \mathrm{M} \mathrm{KCl} \mathrm{[40],} \mathrm{and} \mathrm{the} \mathrm{extract} \mathrm{was} \mathrm{examined} \mathrm{by} \mathrm{an} \mathrm{inductively} \mathrm{coupled}$ plasma optical emission spectrometry (ICP-OES). In a plastic vial, $5 \mathrm{~g}$ of soil was added with $50 \mathrm{~mL}$ of $1 \mathrm{M} \mathrm{KCl}$, followed by shaking for $30 \mathrm{~min}$, and the supernatant was separated by the Whatman no. 42 filter paper. Mehlich No. 1 double acid was used to extract the sample to examine the extractable $\mathrm{Fe}$ and $\mathrm{Mn}$, and the concentration was determined using an atomic absorption spectrometer (AAS) [37]. Next, $2 \mathrm{~g}$ of soil was extracted by the extracting solution of $0.03 \mathrm{~N}$ ammonium fluoride $\left(\mathrm{NH}_{4} \mathrm{~F}\right)$ and $0.1 \mathrm{~N}$ hydrochloric acids $(\mathrm{HCl})$ solution [41], and the concentration was determined by inductively coupled plasma optical emission spectroscopy (ICP-OES, PerkinElmer, Inc., Waltham, MA, USA). Soil inorganic $\mathrm{N}\left(\mathrm{NH}_{4}{ }^{+}-\mathrm{N}\right.$ and $\left.\mathrm{NO}_{3}{ }^{-}-\mathrm{N}\right)$ was analyzed by extracting with a 1:4 ratio of fresh soil: $2 \mathrm{M} \mathrm{KCl}$ phenylmercuric acetate (KCl-PMA) mixture [42] and determined by titration against $0.01 \mathrm{~N} \mathrm{HCl}$.

\subsection{Biochar Analysis}

The $\mathrm{pH}$ of the rice husk biochar was measured using a $\mathrm{pH}$ meter by taking a 1:2.5 ratio of air-dried biochar sample to distilled water [43]. Total $\mathrm{N}$ and total $\mathrm{C}$ in the biochar were analyzed by a CNS analyzer (TrueMac CNS Analyser). $1 \mathrm{M} \mathrm{NH}_{4} \mathrm{OAc}$ buffered solution at $\mathrm{pH} 7$ was used for determining biochar CEC and exchangeable cations [39]. The extracts' $\mathrm{K}, \mathrm{Ca}$, and $\mathrm{Mg}$ were measured by atomic absorption spectrophotometry (PerkinElmer, Inc., Waltham, MA, USA). For total P determination, the dry ashing method followed by ICP-OES was used. A dry combustion method was used to measure the ash content of rice husk biochar. Five g of RHB sample was taken in a crucible and heated at $500{ }^{\circ} \mathrm{C}$ for 
$8 \mathrm{~h}$ [44]. After cooling the crucible to room temperature, it was reweighed. The ash content percentage was then calculated as:

$$
\text { Ash content }(\%)=\frac{\text { Weight of ash }(\mathrm{g})}{\text { The dry mass of biochar }(\mathrm{g})} \times 100,
$$

\subsection{Plant Material Analysis}

In this experiment, the maize variety, $F_{1}$ Hybrid Sweet Corn, was used as a test crop collected from a local market. It is a common variety that Malaysian farmers regularly use. Plant height was determined using a measuring tape at harvest (75 DAS) by taking the average plant height from the base to the tip of the longest leaf [45]. The leaf chlorophyll content was measured using a portable chlorophyll meter (SPAD-502 Konica Minolta, Inc., Tokyo, Japan). The SPAD readings were taken from fully expanded leaves of each plant and averaged for each pot [46]. After harvest, the plant parts (stem and leaves) were placed into envelopes and dried in an oven at $60{ }^{\circ} \mathrm{C}$ for $72 \mathrm{~h}$ [47]. The dried plant parts were ground using a blender, and $0.25 \mathrm{~g}$ was used for the digestion process. The single dry ashing method [48] was used to extract the macro elements of the plant tissues. The concentrations of $\mathrm{P}, \mathrm{K}, \mathrm{Ca}$, and $\mathrm{Mg}$ were determined using an atomic absorption spectrometer (AAS PerkinElmer PinAAcle, 900T, Waltham, MA, USA). Total N of the plant was determined by the TrueMac CNS analyzer (LECO Corporation, St. Joseph, MI, USA). The maize nutrient uptake was calculated by multiplying with the respective dry weight (oven-dry weight) of the plant part with the nutrient content [49].

$$
\text { Uptake }=\text { Total nutrient concentration } \times \text { biomass, }
$$

A number of yield components such as fresh biomass (g), dry biomass (g), cob length $(\mathrm{cm})$, no. of grain per cob, fresh cob weight $(\mathrm{g})$, root length $(\mathrm{cm})$, etc., were measured. Phosphorus use efficiency was calculated by the following equation [50]:

$$
\text { Phosphorus use efficiency (PUE), }\left(\mathrm{kg} \mathrm{ha}^{-1}\right)=(\mathrm{YF}-\mathrm{Yo}) / \mathrm{F} \text {, }
$$

where YF denotes the $\mathrm{P}$ uptake under fertilized treatment $\left(\mathrm{kg} \mathrm{ha}^{-1}\right)$; Yo denotes the $\mathrm{P}$ uptake under control treatment $\left(\mathrm{kg} \mathrm{ha}^{-1}\right)$, and $\mathrm{F}$ indicates the rate of $\mathrm{P}$ applied in that particular treatment $\left(\mathrm{kg} \mathrm{ha}^{-1}\right)$.

\subsection{Soil $\mathrm{CO}_{2}$ Flux Emission Measurement}

A portable LI-8100 automated soil $\mathrm{CO}_{2}$ flux system (LI-COR Biosciences, Lincoln, NE, USA) was used to measure Soil $\mathrm{CO}_{2}$ gas flux on days $1,2,3,4,5,6,7,10,15,20,25,30$, $35,40,45,50,55,60,65,70$, and 75. Each day, the data was taken between 9.00 a.m. to 11.00 a.m. [51]. To minimize the soil surface disturbance, a polyvinyl chloride (PVC) pipe $(11.5 \mathrm{~cm}$ in height and $20 \mathrm{~cm}$ inner diameter) was inserted 7 to $8 \mathrm{~cm}$ into the soil on the previous day of the first measurement. All the PVC collars were kept fixed during the whole experiment. The cumulative soil $\mathrm{CO}_{2}$ flux measurement was calculated by linear interpolation [52].

\subsection{Percent Relative Data}

The relative data of the value were expressed as percentages, relative to control for each element recommended by Ashraf et al. [53]:

$$
\text { Relative data }(\%)=\frac{\text { Treatment value }- \text { control value }}{\text { control value }} \times 100
$$

where the treatment value was the rice husk biochar and lime amended treatment, and the control value was without amendment. 


\subsection{Statistical Analysis}

All data were analyzed using the analysis of variance (ANOVA) procedure, and means were separated by Tukey's Honestly Significant Difference (HSD) test at a 5\% level of confidence using Statistical Analysis System, version: SAS 9.4 software (SAS Institute Inc., Cary, NC, USA). In addition, linear regression and principal component analysis were also performed to understand the relationships and contribution of different factors on the yield of maize.

\section{Results}

\subsection{Effect of Treatments on Changes in Nutrients of the Post-Harvest Soil}

The effect of RHB, dolomitic limestone, and different doses of $\mathrm{P}$ application on soil nutrient availability of maize are presented in Table 4 . The addition of treatments significantly altered soil $\mathrm{pH}$. The soil $\mathrm{pH}$ noted ranged from 4.49 to 6.14 . The lowest $\mathrm{pH}$ (4.49) was observed from the control treatment. The treatment with $100 \%$ lime $+10 \mathrm{tha}^{-1} \mathrm{RHB}+$ $100 \%$ TSP $\left(\mathrm{T}_{3}\right)$ showed the highest soil $\mathrm{pH}$, which was increased by 1.65 units compared to control (6.14-4.49), although $\mathrm{T}_{3}$ and $\mathrm{T}_{5}$ led the same statistical value.

It was observed that soil available $P$ after harvest of the maize crop was significantly increased using RHB, lime, and P application (Table 4). The available P examined ranged from 5.43 to $14.05 \mathrm{mg} \mathrm{kg}^{-1}$ soil. The maximum $\mathrm{P}$ content found from $\mathrm{T}_{9}(75 \%$ lime + $15 \mathrm{tha}^{-1} \mathrm{RHB}+100 \%$ TSP), followed by $\mathrm{T}_{3}\left(100 \%\right.$ lime $+10 \mathrm{tha}{ }^{-1} \mathrm{RHB}+100 \%$ TSP $)$ and $\mathrm{T}_{6}$ (75\% lime $+10 \mathrm{t} \mathrm{ha}^{-1} \mathrm{RHB}+100 \% \mathrm{TSP}$ ) and the minimum found from control treatment $\left(\mathrm{T}_{1}\right)$. The highest increment of available $\mathrm{P}$ was $158.75 \%$ from $\mathrm{T}_{3}$ compared to the control. The application of soil amendments significantly increased soil exchangeable K shown in Table 4 . The highest $\mathrm{K}$ content $\left(1.49 \mathrm{cmol}_{\mathrm{c}} \mathrm{kg}^{-1}\right)$ observed from $\mathrm{T}_{9}\left(75 \%\right.$ lime $+15 \mathrm{tha}^{-1}$ RHB $+100 \%$ TSP), followed by $\mathrm{T}_{6}\left(75 \%\right.$ lime $+10 \mathrm{t} \mathrm{ha}^{-1} \mathrm{RHB}+100 \%$ TSP). The lowest $\mathrm{K}$ content $\left(0.34 \mathrm{cmol}_{\mathrm{c}} \mathrm{kg}^{-1}\right)$ was analyzed from Control $\left(\mathrm{T}_{1}\right)$. The maximum increment of exchangeable $\mathrm{K}$ was noted $338.24 \%$ from $\mathrm{T}_{6}$ compared over control treatment.

It was observed that soil exchangeable Ca was significantly increased using RHB, lime, and $\mathrm{P}$ application (Table 4). The maximum exchangeable Ca $4.15 \mathrm{cmol}_{\mathrm{C}} \mathrm{kg}^{-1}$ found from $\mathrm{T}_{9}$ (75\% lime $\left.+15 \mathrm{tha}^{-1} \mathrm{RHB}+100 \% \mathrm{TSP}\right)$, followed by $\mathrm{T}_{10}\left(75 \%\right.$ lime $+15 \mathrm{tha}^{-1} \mathrm{RHB}+75 \%$ TSP) and the minimum exchangeable Ca $1.43 \mathrm{cmol}_{\mathrm{C}} \mathrm{kg}^{-1}$ found from control treatment $\left(\mathrm{T}_{1}\right)$. The exchangeable Ca was highest increased by $190.21 \%$ from $\mathrm{T}_{9}$ compared to the untreated soil. The exchangeable $\mathrm{Mg}$ of soil under this study was significantly influenced by the amendments represented in Table 4 . The highest $\mathrm{Mg}$ content $\left(1.47 \mathrm{cmol}_{\mathrm{C}} \mathrm{kg}^{-1}\right)$ observed from $\mathrm{T}_{3}\left(100 \%\right.$ lime $\left.+10 \mathrm{tha}^{-1} \mathrm{RHB}+100 \% \mathrm{TSP}\right)$, followed by $\mathrm{T}_{9}\left(75 \%\right.$ lime $+15 \mathrm{tha}^{-1}$ RHB $+100 \%$ TSP). The lowest $\mathrm{Mg}$ content $\left(0.44 \mathrm{cmol}_{\mathrm{C}} \mathrm{kg}^{-1}\right)$ was analyzed from Control $\left(\mathrm{T}_{1}\right)$. The maximum increment of exchangeable $\mathrm{Mg}$ was $234.09 \%$ from $\mathrm{T}_{3}$ compared to the control treatment.

The exchangeable $\mathrm{Al}$ in soil was significantly influenced by the treatments presented in Table 4. The value of exchangeable Al ranged from 0.08 to $2.53 \mathrm{cmol}_{\mathrm{c}} \mathrm{kg}^{-1}$ soil. $\mathrm{T}_{3}(100 \%$ lime $+10 \mathrm{t} \mathrm{ha}^{-1} \mathrm{RHB}+100 \%$ TSP) noted the lowest value $\left(0.08 \mathrm{cmol}_{\mathrm{c}} \mathrm{kg}^{-1}\right)$, followed by $\mathrm{T}_{5}\left(100 \%\right.$ lime $\left.+10 \mathrm{tha}^{-1} \mathrm{RHB}+50 \% \mathrm{TSP}\right)$. The highest value $\left(2.53 \mathrm{cmol}_{\mathrm{C}} \mathrm{kg}^{-1}\right)$ was found from the control treatment. It was observed that soil-extractable Fe was significantly decreased using RHB, lime, and P application in maize crops (Table 4). The value of extractable Fe was significantly different, with the values ranging from 43.36 to $80.05 \mathrm{mg} \mathrm{kg}^{-1}$. The lowest value ( $\left.43.36 \mathrm{mg} \mathrm{kg}^{-1}\right)$ of Fe was noted from $\mathrm{T}_{7}\left(75 \%\right.$ lime $+10 \mathrm{tha}^{-1} \mathrm{RHB}+$ $75 \%$ TSP), and this value decreased by $45.83 \%$ compared to the control. The highest value (80.05 $\mathrm{mg} \mathrm{kg}^{-1}$ ) was observed by the control $\left(\mathrm{T}_{1}\right)$ treatment. The extractable Mn of soil under this study was significantly influenced by the amendments represented in Table 4 . $\mathrm{T}_{3}\left(100 \%\right.$ lime $\left.+10 \mathrm{tha}^{-1} \mathrm{RHB}+100 \% \mathrm{TSP}\right)$ showed the highest Mn content $\left(6.58 \mathrm{mg} \mathrm{kg}^{-1}\right)$, followed by $\mathrm{T}_{9}\left(75 \%\right.$ lime $\left.+15 \mathrm{tha}^{-1} \mathrm{RHB}+100 \% \mathrm{TSP}\right)$. The control treatment $\left(\mathrm{T}_{1}\right)$ showed the lowest $\mathrm{Mn}$ content $\left(4.67 \mathrm{mg} \mathrm{kg}^{-1}\right)$. The maximum increment of extractable $\mathrm{Mn}$ was $40.90 \%$ from $\mathrm{T}_{3}$ compared to control. 
Table 4. Effect of treatments on changes in nutrients of the post-harvest soil.

\begin{tabular}{|c|c|c|c|c|c|c|c|c|}
\hline Treatment & $\mathrm{pH}$ & $\begin{array}{c}\text { Available P } \\
\left(\mathrm{mg} \mathrm{kg}^{-1}\right)\end{array}$ & $\begin{array}{c}\text { Exchangeable K } \\
\left(\mathrm{cmol}_{\mathrm{c}} \mathrm{kg}^{-1}\right)\end{array}$ & $\begin{array}{c}\text { Exchangeable Ca } \\
\left(\mathrm{cmol}_{\mathrm{c}} \mathrm{kg}^{-1}\right)\end{array}$ & $\begin{array}{l}\text { Exchangeable } \mathrm{Mg} \\
\quad\left(\mathrm{cmol}_{\mathrm{c}} \mathrm{kg}^{-1}\right)\end{array}$ & $\begin{array}{c}\text { Exchangeable Al } \\
\left(\mathrm{cmol}_{\mathrm{c}} \mathrm{kg}^{-1}\right)\end{array}$ & $\begin{array}{c}\text { Extractable Fe } \\
\left(\mathrm{mg} \mathrm{kg}^{-1}\right)\end{array}$ & $\begin{array}{c}\text { Extractable Mn } \\
\left(\mathrm{mg} \mathrm{kg}^{-1}\right)\end{array}$ \\
\hline $\mathrm{T}_{1}$ & $4.49^{\mathrm{e}} \pm 0.036$ & $5.43^{h} \pm 0.017$ & $0.34^{\mathrm{e}} \pm 0.022$ & $1.43^{g} \pm 0.013$ & $0.44^{g} \pm 0.012$ & $2.53^{a} \pm 0.013$ & $80.05^{\mathrm{a}} \pm 1.56$ & $4.67^{\mathrm{f}} \pm 0.041$ \\
\hline $\mathrm{T}_{2}$ & $4.55^{\mathrm{e}} \pm 0.039$ & $5.85^{\mathrm{g}} \pm 0.015$ & $0.47^{\mathrm{d}} \pm 0.025$ & $1.60^{\mathrm{f}} \pm 0.013$ & $0.55^{\mathrm{g}} \pm 0.025$ & $2.48^{\mathrm{a}} \pm 0.021$ & $84.09^{\mathrm{a}} \pm 1.31$ & $4.71^{\mathrm{f}} \pm 0.016$ \\
\hline $\mathrm{T}_{3}$ & $6.14^{\mathrm{a}} \pm 0.023$ & $13.56^{\mathrm{b}} \pm 0.206$ & $1.37^{b c} \pm 0.015$ & $4.05^{b} \pm 0.013$ & $1.47^{\mathrm{a}} \pm 0.015$ & $0.08^{\mathrm{f}} \pm 0.011$ & $51.66^{\mathrm{bc}} \pm 1.14$ & $6.58^{a} \pm 0.016$ \\
\hline $\mathrm{T}_{4}$ & $5.91^{\mathrm{d}} \pm 0.013$ & $11.41^{\mathrm{c}} \pm 0.078$ & $1.34^{\mathrm{c}} \pm 0.019$ & $3.97^{b c} \pm 0.015$ & $1.31 b^{c d} \pm 0.019$ & $0.18^{\mathrm{d}} \pm 0.015$ & $47.25^{\mathrm{cd}} \pm 1.01$ & $6.11^{\mathrm{c}} \pm 0.025$ \\
\hline $\mathrm{T}_{5}$ & $6.10^{\mathrm{ab}} \pm 0.013$ & $8.74^{\mathrm{e}} \pm 0.029$ & $1.35^{b c} \pm 0.018$ & $3.96^{\mathrm{cd}} \pm 0.021$ & $1.19^{\mathrm{f}} \pm 0.016$ & $0.09^{\text {ef }} \pm 0.018$ & $55.03^{b} \pm 1.06$ & $5.84^{\mathrm{e}} \pm 0.021$ \\
\hline $\mathrm{T}_{6}$ & $5.93^{\mathrm{cd}} \pm 0.015$ & $13.44^{\mathrm{b}} \pm 0.048$ & $1.43^{\mathrm{ab}} \pm 0.018$ & $3.84^{\mathrm{e}} \pm 0.023$ & $1.33^{b c} \pm 0.013$ & $0.24^{\mathrm{d}} \pm 0.019$ & $45.86^{\mathrm{d}} \pm 0.70$ & $6.27^{b} \pm 0.022$ \\
\hline $\mathrm{T}_{7}$ & $6.02^{\mathrm{cb}} \pm 0.019$ & $11.01^{\mathrm{d}} \pm 0.065$ & $1.38^{b c} \pm 0.015$ & $4.05^{b} \pm 0.017$ & $1.26^{\text {de }} \pm 0.019$ & $0.12^{\mathrm{ef}} \pm 0.017$ & $43.36^{\mathrm{d}} \pm 1.50$ & $6.08^{c} \pm 0.015$ \\
\hline $\mathrm{T}_{8}$ & $5.95^{\mathrm{cd}} \pm 0.017$ & $8.05^{\mathrm{f}} \pm 0.035$ & $1.39^{b c} \pm 0.013$ & $3.81^{\mathrm{e}} \pm 0.022$ & $1.22 e^{\text {ef }} \pm 0.024$ & $0.17^{\text {de }} \pm 0.018$ & $54.46^{\mathrm{b}} \pm 1.48$ & $5.95^{\mathrm{de}} \pm 0.019$ \\
\hline $\mathrm{T}_{9}$ & $5.90^{\mathrm{d}} \pm 0.015$ & $14.05^{\mathrm{a}} \pm 0.033$ & $1.49^{\mathrm{a}} \pm 0.017$ & $4.15^{\mathrm{a}} \pm 0.011$ & $1.46^{\mathrm{a}} \pm 0.013$ & $0.39^{b c} \pm 0.016$ & $46.49^{\mathrm{cd}} \pm 1.28$ & $6.48^{\mathrm{a}} \pm 0.019$ \\
\hline $\mathrm{T}_{10}$ & $5.87^{\mathrm{d}} \pm 0.026$ & $11.33^{\mathrm{cd}} \pm 0.043$ & $1.34^{\mathrm{c}} \pm 0.018$ & $4.14^{\mathrm{a}} \pm 0.013$ & $1.34^{\mathrm{b}} \pm 0.019$ & $0.44^{\mathrm{b}} \pm 0.016$ & $47.21^{\mathrm{cd}} \pm 1.28$ & $6.27^{b} \pm 0.022$ \\
\hline $\mathrm{T}_{11}$ & $5.92^{\mathrm{cd}} \pm 0.013$ & $8.73^{e} \pm 0.042$ & $1.39 \mathrm{cb} \pm 0.016$ & 3.88 de \pm 0.026 & $1.28^{\mathrm{cd}} \pm 0.019$ & $0.34^{\mathrm{c}} \pm 0.024$ & $53.46^{\mathrm{b}} \pm 0.78$ & $6.05 \mathrm{~cd} \pm 0.018$ \\
\hline$p$-value & $<0.0001$ & $<0.0001$ & $<0.0001$ & $<0.0001$ & $<0.0001$ & $<0.0001$ & $<0.0001$ & $<0.0001$ \\
\hline
\end{tabular}

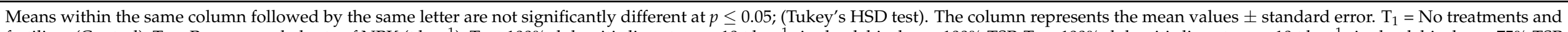

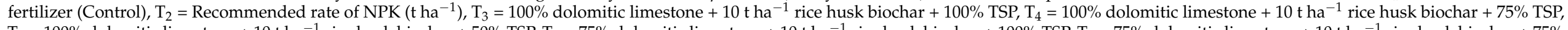

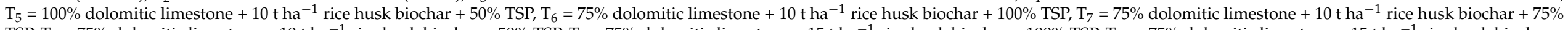

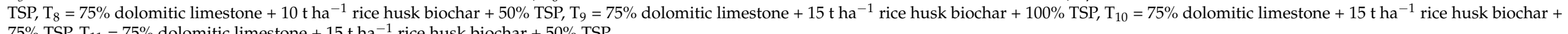
$75 \%$ TSP, $\mathrm{T}_{11}=75 \%$ dolomitic limestone $+15 \mathrm{tha}^{-1}$ rice husk biochar $+50 \%$ TSP. 
The incorporation of RHB, lime, and P fertilizer contribute to a greater reduction in soil exchangeable acidity compared with the control represented in Figure 1 . The lowest value $\left(0.12 \mathrm{cmol}_{\mathrm{C}} \mathrm{kg}^{-1}\right)$ of exchangeable acidity was noted from $\mathrm{T}_{3}\left(100 \%\right.$ lime $+10 \mathrm{tha}^{-1}$ RHB $+100 \%$ TSP), which decreased by $95.20 \%$. The maximum soil exchangeable acidity $\left(2.50 \mathrm{cmol}_{\mathrm{C}} \mathrm{kg}^{-1}\right)$ was observed from the control.

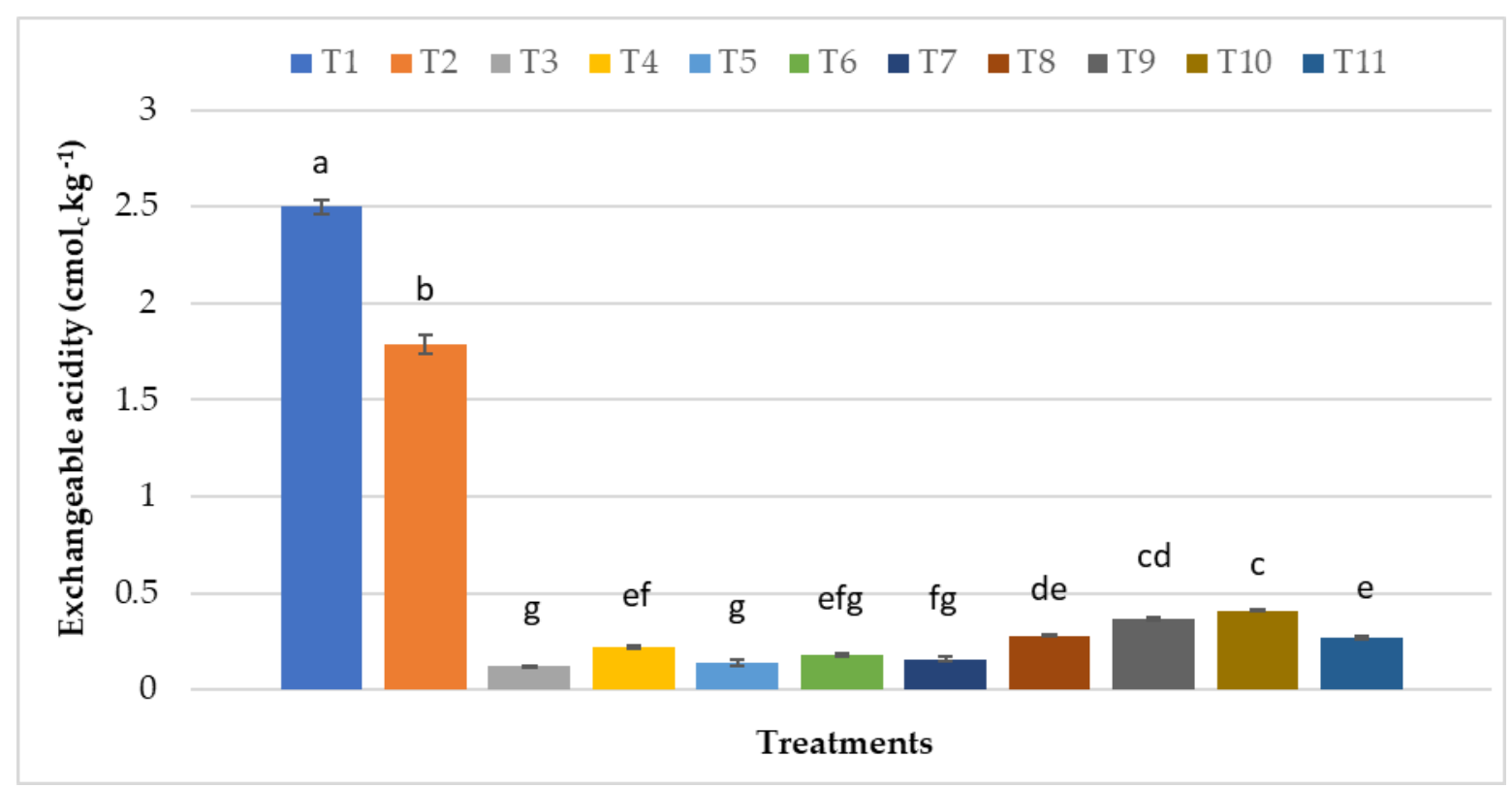

Figure 1. Effect of treatments on changes in soil exchangeable acidity of the post-harvest soil. Bar errors show \pm standard error of four replications. $\mathrm{T}_{1}=\mathrm{No}$ treatments and fertilizer (Control), $\mathrm{T}_{2}=$ Recommended rate of NPK $\left(\mathrm{t} \mathrm{ha}{ }^{-1}\right), \mathrm{T}_{3}=100 \%$ dolomitic limestone $+10 \mathrm{t} \mathrm{ha}^{-1}$ rice husk biochar $+100 \% \mathrm{TSP}, \mathrm{T}_{4}=100 \%$ dolomitic limestone $+10 \mathrm{t} \mathrm{ha}^{-1}$ rice husk biochar + $75 \% \mathrm{TSP}, \mathrm{T}_{5}=100 \%$ dolomitic limestone $+10 \mathrm{t} \mathrm{ha}^{-1}$ rice husk biochar $+50 \% \mathrm{TSP}, \mathrm{T}_{6}=75 \%$ dolomitic limestone $+10 \mathrm{t}$ ha ${ }^{-1}$ rice husk biochar $+100 \% \mathrm{TSP}, \mathrm{T}_{7}=75 \%$ dolomitic limestone $+10 \mathrm{tha}{ }^{-1}$ rice husk biochar $+75 \% \mathrm{TSP}, \mathrm{T}_{8}=75 \%$ dolomitic limestone $+10 \mathrm{t} \mathrm{ha}^{-1}$ rice husk biochar $+50 \% \mathrm{TSP}, \mathrm{T}_{9}=75 \%$ dolomitic limestone $+15 \mathrm{tha}^{-1}$ rice husk biochar $+100 \% \mathrm{TSP}$, $\mathrm{T}_{10}=75 \%$ dolomitic limestone $+15 \mathrm{t} \mathrm{ha}^{-1}$ rice husk biochar $+75 \% \mathrm{TSP}, \mathrm{T}_{11}=75 \%$ dolomitic limestone $+15 \mathrm{t}$ ha ${ }^{-1}$ rice husk biochar $+50 \%$ TSP.

\subsection{Effect of Treatments on Changes in Plant Growth and Yield Contributing Characters}

Maize plant height, dry biomass, root length, and leaf chlorophyll content (SPAD value) were significantly increased by adding RHB, lime, and $P$ fertilizers at harvest, presented in Table 5. For example, $\mathrm{T}_{9}\left(75 \%\right.$ lime $\left.+15 \mathrm{tha}^{-1} \mathrm{RHB}+100 \% \mathrm{TSP}\right)$ showed the highest plant height of $230.45 \mathrm{~cm}$, while $\mathrm{T}_{1}$ exhibited the lowest height of $179.20 \mathrm{~cm}$; though $\mathrm{T}_{3}$ to $\mathrm{T}_{11}$ led to the same statistical difference.

There was a significant alteration in dry biomass, root length, and SPAD value of maize after applying the treatments compared to the unamended soil (Table 5). The maximum dry biomass of $84.31 \mathrm{~g}$ was noted from $\mathrm{T}_{6}\left(75 \%\right.$ lime $\left.+10 \mathrm{tha}^{-1} \mathrm{RHB}+100 \% \mathrm{TSP}\right)$, while the lowest dry biomass $(22.32 \mathrm{~g})$ was found from the control treatment $\left(\mathrm{T}_{1}\right)$. The highest root length of $79.61 \mathrm{~cm}$ was from $\mathrm{T}_{7}\left(75 \%\right.$ lime $\left.+10 \mathrm{tha}^{-1} \mathrm{RHB}+75 \% \mathrm{TSP}\right)$. In contrast, the lowest root length $(46.94 \mathrm{~cm})$ was revealed from the control $\left(\mathrm{T}_{1}\right) . \mathrm{T}_{5}\left(100 \%\right.$ lime $+10 \mathrm{tha}^{-1}$ RHB $+50 \%$ TSP) showed the highest SPAD value of 52.03, while the lowest (40.70) was from untreated soil $\left(\mathrm{T}_{1}\right)$. Although, $\mathrm{T}_{3}$ to $\mathrm{T}_{11}$ revealed the same statistical differences in terms of dry biomass, root length, and leaf chlorophyll content. The highest increase in the SPAD value was $27.84 \%$ from $\mathrm{T}_{5}\left(100 \%\right.$ lime $\left.+10 \mathrm{tha}^{-1} \mathrm{RHB}+50 \% \mathrm{TSP}\right)$ compared to control.

The maize cob length, fresh cob weight, no. of grain per cob, and yield were significantly changed (Table 6) by combined application of RHB, lime, and P fertilizer ( $T_{3}$ to $T_{11}$ ) compared to that of untreated soil $\left(\mathrm{T}_{1}\right.$ and $\left.\mathrm{T}_{2}\right)$. The highest cob length $(23.75 \mathrm{~cm})$, fresh cob 
weight (290.60 g), and no. of grain per cob (635) showed by $\mathrm{T}_{6}\left(75 \%\right.$ lime $+10 \mathrm{t} \mathrm{ha}^{-1} \mathrm{RHB}$ $+100 \%$ TSP), while the control treatment showed the lowest of all. However, from $\mathrm{T}_{3}$ to $\mathrm{T}_{11}$ noted as statistically similar in cob length and no. of grain per cob; $\mathrm{T}_{3}, \mathrm{~T}_{4}, \mathrm{~T}_{5}, \mathrm{~T}_{6}, \mathrm{~T}_{7}$, $\mathrm{T}_{8}, \mathrm{~T}_{10}$, and $\mathrm{T}_{11}$ showed the same statistical value of fresh cob weight. The highest yield of $15.50 \mathrm{t} \mathrm{ha}^{-1}$ was exhibited by $\mathrm{T}_{6}\left(75 \%\right.$ lime $\left.+10 \mathrm{t} \mathrm{ha}^{-1} \mathrm{RHB}+100 \% \mathrm{TSP}\right)$ followed by $\mathrm{T}_{4}\left(100 \%\right.$ lime $\left.+10 \mathrm{t} \mathrm{ha}^{-1} \mathrm{RHB}+75 \% \mathrm{TSP}\right)$; although $\mathrm{T}_{3}, \mathrm{~T}_{4}, \mathrm{~T}_{5}, \mathrm{~T}_{6}, \mathrm{~T}_{7}, \mathrm{~T}_{8}, \mathrm{~T}_{10}$, and $\mathrm{T}_{11}$ showed the similar statistical value. The control treatment found a minimum crop yield of $9.22 \mathrm{tha}^{-1}$.

Table 5. Effect of treatments on plant growth.

\begin{tabular}{ccccc}
\hline Treatment & $\begin{array}{c}\text { Plant Height } \\
\mathbf{( c m )}\end{array}$ & $\begin{array}{c}\text { Dry Biomass } \\
\mathbf{( g )}\end{array}$ & $\begin{array}{c}\text { Root Length } \\
\mathbf{( c m})\end{array}$ & SPAD Value \\
\hline $\mathrm{T}_{1}$ & $179.20^{\mathrm{c}} \pm 1.86$ & $22.32^{\mathrm{c}} \pm 1.82$ & $46.94^{\mathrm{b}} \pm 1.21$ & $40.70^{\mathrm{c}} \pm 0.684$ \\
$\mathrm{~T}_{2}$ & $195.07^{\mathrm{b}} \pm 1.53$ & $39.37^{\mathrm{b}} \pm 2.21$ & $53.65^{\mathrm{b}} \pm 1.00$ & $43.70^{\mathrm{bc}} \pm 0.62$ \\
$\mathrm{~T}_{3}$ & $225.66^{\mathrm{a}} \pm 2.86$ & $84.01^{\mathrm{a}} \pm 1.90$ & $73.01^{\mathrm{a}} \pm 1.67$ & $50.45^{\mathrm{a}} \pm 0.924$ \\
$\mathrm{~T}_{4}$ & $224.52^{\mathrm{a}} \pm 4.06$ & $79.23^{\mathrm{a}} \pm 1.93$ & $76.16^{\mathrm{a}} \pm 1.25$ & $49.03^{\mathrm{a}} \pm 0.578$ \\
$\mathrm{~T}_{5}$ & $225.30^{\mathrm{a}} \pm 2.89$ & $80.03^{\mathrm{a}} \pm 1.84$ & $77.18^{\mathrm{a}} \pm 1.61$ & $52.03^{\mathrm{a}} \pm 0.657$ \\
$\mathrm{~T}_{6}$ & $222.26^{\mathrm{a}} \pm 2.53$ & $84.31^{\mathrm{a}} \pm 1.82$ & $77.35^{\mathrm{a}} \pm 1.94$ & $50.75^{\mathrm{a}} \pm 1.372$ \\
$\mathrm{~T}_{7}$ & $223.92^{\mathrm{a}} \pm 2.20$ & $81.87^{\mathrm{a}} \pm 2.05$ & $79.61^{\mathrm{a}} \pm 4.09$ & $50.68^{\mathrm{a}} \pm 1.148$ \\
$\mathrm{~T}_{8}$ & $224.86^{\mathrm{a}} \pm 1.98$ & $75.37^{\mathrm{a}} \pm 2.09$ & $75.23^{\mathrm{a}} \pm 2.35$ & $48.95^{\mathrm{ab}} \pm 1.383$ \\
$\mathrm{~T}_{9}$ & $230.45^{\mathrm{a}} \pm 2.22$ & $78.91^{\mathrm{a}} \pm 1.77$ & $71.95^{\mathrm{a}} \pm 1.84$ & $49.78^{\mathrm{a}} \pm 1.193$ \\
$\mathrm{~T}_{10}$ & $226.07^{\mathrm{a}} \pm 1.81$ & $81.62^{\mathrm{a}} \pm 1.95$ & $73.24^{\mathrm{a}} \pm 2.26$ & $49.63^{\mathrm{a}} \pm 1.497$ \\
$\mathrm{~T}_{11}$ & $222.91^{\mathrm{a}} \pm 1.81$ & $77.45^{\mathrm{a}} \pm 2.15$ & $76.90^{\mathrm{a}} \pm 2.06$ & $50.88^{\mathrm{a}} \pm 1.13$ \\
$p$-value & $<0.0001$ & $<0.0001$ & $<0.0001$ & $<0.0001$ \\
\hline
\end{tabular}

Means within the same column followed by the same letter are not significantly different at $p \leq 0.05$; (Tukey's HSD test). The column represents the mean values \pm standard error. $\mathrm{T}_{1}=$ No treatments and fertilizer (Control) $\mathrm{T}_{2}=$ Recommended rate of NPK $\left(\mathrm{t} \mathrm{ha}^{-1}\right), \mathrm{T}_{3}=100 \%$ dolomitic limestone $+10 \mathrm{tha}^{-1}$ rice husk biochar $+100 \%$ TSP, $\mathrm{T}_{4}=100 \%$ dolomitic limestone $+10 \mathrm{tha}^{-1}$ rice husk biochar $+75 \% \mathrm{TSP}, \mathrm{T}_{5}=100 \%$ dolomitic limestone + $10 \mathrm{t} \mathrm{ha}^{-1}$ rice husk biochar $+50 \% \mathrm{TSP}, \mathrm{T}_{6}=75 \%$ dolomitic limestone $+10 \mathrm{t} \mathrm{ha}^{-1}$ rice husk biochar $+100 \%$ TSP $\mathrm{T}_{7}=75 \%$ dolomitic limestone $+10 \mathrm{t} \mathrm{ha}^{-1}$ rice husk biochar $+75 \% \mathrm{TSP}, \mathrm{T}_{8}=75 \%$ dolomitic limestone $+10 \mathrm{t} \mathrm{ha}^{-1}$ rice husk biochar $+50 \%$ TSP, $\mathrm{T}_{9}=75 \%$ dolomitic limestone $+15 \mathrm{t} \mathrm{ha}^{-1}$ rice husk biochar $+100 \% \mathrm{TSP}, \mathrm{T}_{10}=75 \%$ dolomitic limestone $+15 \mathrm{t} \mathrm{ha}^{-1}$ rice husk biochar $+75 \%$ TSP, $\mathrm{T}_{11}=75 \%$ dolomitic limestone $+15 \mathrm{tha}^{-1}$ rice husk biochar $+50 \%$ TSP.

Table 6. Effect of treatments on yield contributing characters.

\begin{tabular}{ccccc}
\hline Treatment & $\begin{array}{c}\text { Cob Length } \\
(\mathbf{c m})\end{array}$ & $\begin{array}{c}\text { Fresh Cob Weight } \\
\mathbf{( g )}\end{array}$ & $\begin{array}{c}\text { No. of Grain } \\
\text { per Cob }\end{array}$ & $\begin{array}{c}\text { Yield } \\
\left(\mathbf{t ~ h a} \mathbf{~}^{-\mathbf{1}} \mathbf{)}\right.\end{array}$ \\
\hline $\mathrm{T}_{1}$ & $18.28^{\mathrm{a}} \pm 0.559$ & $172.98^{\mathrm{c}} \pm 1.69$ & $323^{\mathrm{d}} \pm 5.82$ & $9.22^{\mathrm{c}} \pm 0.090$ \\
$\mathrm{~T}_{2}$ & $19.50^{\mathrm{b}} \pm 0.507$ & $183.15^{\mathrm{c}} \pm 2.33$ & $480^{\mathrm{c}} \pm 4.19$ & $9.77^{\mathrm{c}} \pm 0.126$ \\
$\mathrm{~T}_{3}$ & $23.18^{\mathrm{a}} \pm 0.332$ & $286.68^{\mathrm{ab}} \pm 2.31$ & $624^{\mathrm{ab}} \pm 5.18$ & $15.29^{\mathrm{ab}} \pm 0.122$ \\
$\mathrm{~T}_{4}$ & $22.50^{\mathrm{a}} \pm 0.492$ & $290.03^{\mathrm{a}} \pm 2.27$ & $622^{\mathrm{ab}} \pm 4.19$ & $15.47^{\mathrm{a}} \pm 0.119$ \\
$\mathrm{~T}_{5}$ & $22.48^{\mathrm{a}} \pm 0.496$ & $286.73^{\mathrm{ab}} \pm 3.39$ & $628^{\mathrm{ab}} \pm 4.21$ & $15.29^{\mathrm{ab}} \pm 0.181$ \\
$\mathrm{~T}_{6}$ & $23.75^{\mathrm{a}} \pm 0.247$ & $290.60^{\mathrm{a}} \pm 2.21$ & $635^{\mathrm{a}} \pm 3.86$ & $15.50^{\mathrm{a}} \pm 0.119$ \\
$\mathrm{~T}_{7}$ & $23.15^{\mathrm{a}} \pm 0.184$ & $279.45^{\mathrm{ab}} \pm 2.53$ & $630^{\mathrm{ab}} \pm 5.21$ & $14.90^{\mathrm{ab}} \pm 0.136$ \\
$\mathrm{~T}_{8}$ & $22.45^{\mathrm{a}} \pm 0.403$ & $279.43^{\mathrm{ab}} \pm 2.05$ & $611^{\mathrm{b}} \pm 5.05$ & $14.90^{\mathrm{ab}} \pm 0.110$ \\
$\mathrm{~T}_{9}$ & $23.10^{\mathrm{a}} \pm 0.381$ & $276.80^{\mathrm{b}} \pm 1.64$ & $622^{\mathrm{ab}} \pm 5.74$ & $14.77^{\mathrm{b}} \pm 0.080$ \\
$\mathrm{~T}_{10}$ & $22.90^{\mathrm{a}} \pm 0.443$ & $287.75^{\mathrm{ab}} \pm 1.48$ & $616^{\mathrm{ab}} \pm 3.38$ & $15.35^{\mathrm{ab}} \pm 0.08$ \\
$\mathrm{~T}_{11}$ & $23.25^{\mathrm{a}} \pm 0.366$ & $282.45^{\mathrm{ab}} \pm 1.90$ & $618^{\mathrm{ab}} \pm 4.91$ & $15.07^{\mathrm{ab}} \pm 0.101$ \\
$p$-value & $<0.0001$ & $<0.0001$ & $<0.0001$ & $<0.0001$ \\
\hline
\end{tabular}

Means within the same column followed by the same letter are not significantly different at $p \leq 0.05$; (Tukey's HSD test). The column represents the mean values \pm standard error. $T_{1}=$ No treatments and fertilizer (Control), $\mathrm{T}_{2}=$ Recommended rate of NPK $\left(\mathrm{tha}^{-1}\right), \mathrm{T}_{3}=100 \%$ dolomitic limestone $+10 \mathrm{tha}{ }^{-1}$ rice husk biochar $+100 \%$ TSP, $\mathrm{T}_{4}=100 \%$ dolomitic limestone $+10 \mathrm{tha}^{-1}$ rice husk biochar $+75 \% \mathrm{TSP}, \mathrm{T}_{5}=100 \%$ dolomitic limestone + $10 \mathrm{tha}^{-1}$ rice husk biochar $+50 \%$ TSP, $\mathrm{T}_{6}=75 \%$ dolomitic limestone $+10 \mathrm{tha}^{-1}$ rice husk biochar $+100 \%$ TSP $\mathrm{T}_{7}=75 \%$ dolomitic limestone $+10 \mathrm{tha}^{-1}$ rice husk biochar $+75 \%$ TSP, $\mathrm{T}_{8}=75 \%$ dolomitic limestone $+10 \mathrm{tha}^{-1}$ rice husk biochar $+50 \% \mathrm{TSP}, \mathrm{T}_{9}=75 \%$ dolomitic limestone $+15 \mathrm{tha}^{-1}$ rice husk biochar $+100 \% \mathrm{TSP}, \mathrm{T}_{10}=75 \%$ dolomitic limestone $+15 \mathrm{t} \mathrm{ha}^{-1}$ rice husk biochar $+75 \%$ TSP, $\mathrm{T}_{11}=75 \%$ dolomitic limestone $+15 \mathrm{t} \mathrm{ha}^{-1}$ rice husk biochar $+50 \%$ TSP. 


\subsection{Effect of Treatments on Changes in Plant Nutrient Concentration and Uptake}

There was a significant difference in plant nutrient concentration and uptake of maize, represented in Tables 7 and 8 . With $\mathrm{T}_{1}$ and $\mathrm{T}_{2}$, the concentration and total uptake of $\mathrm{N}, \mathrm{P}, \mathrm{K}$, $\mathrm{Ca}$, and $\mathrm{Mg}$ taken up by the maize plant were statistically lower than those of biochar and lime amended treatments $\left(\mathrm{T}_{3}\right.$ to $\left.\mathrm{T}_{11}\right)$. The highest uptake of $\mathrm{N}\left(2027.66 \mathrm{mg} \mathrm{plant}^{-1}\right)$ and Ca (526.94 $\mathrm{mg} \mathrm{plant}^{-1}$ ) obtained from $\mathrm{T}_{6}\left(75 \%\right.$ lime $+10 \mathrm{tha}^{-1} \mathrm{RHB}+100 \%$ TSP); the highest $\mathrm{P}$ (250.98 $\mathrm{mg} \mathrm{plant}^{-1}$ ) noted from $\mathrm{T}_{10}\left(75 \%\right.$ lime $\left.+15 \mathrm{t} \mathrm{ha}^{-1} \mathrm{RHB}+75 \% \mathrm{TSP}\right) ;$ but $\mathrm{T}_{6}, \mathrm{~T}_{9}$, and $\mathrm{T}_{10}$ have the same statistical value. The maximum $\mathrm{K}$ uptake (1986.84 $\mathrm{mg} \mathrm{plant}^{-1}$ ), and $\mathrm{Mg}$ uptake (273.03 $\left.\mathrm{mg} \mathrm{plant}^{-1}\right)$ are shown from $\mathrm{T}_{3}\left(100 \%\right.$ lime $+10 \mathrm{t} \mathrm{ha}^{-1} \mathrm{RHB}+100 \%$ TSP). Figure 2 shows the maximum (145.64\%) phosphorus use efficiency (PUE) noted from $\mathrm{T}_{10}\left(75 \%\right.$ lime $\left.+15 \mathrm{t} \mathrm{ha}^{-1} \mathrm{RHB}+75 \% \mathrm{TSP}\right)$ followed by $\mathrm{T}_{6}(133.37 \%)$ and $\mathrm{T}_{9}(131.75 \%)$.

Table 7. Effect of treatments on changes in plant nutrient concentration.

\begin{tabular}{|c|c|c|c|c|c|}
\hline Treatment & $\begin{array}{c}\text { N Concentration } \\
(\%)\end{array}$ & $\begin{array}{c}\text { P Concentration } \\
(\%)\end{array}$ & $\begin{array}{c}\text { K Concentration } \\
(\%)\end{array}$ & $\begin{array}{c}\text { Ca Concentration } \\
(\%)\end{array}$ & $\begin{array}{c}\text { Mg Concentration } \\
(\%)\end{array}$ \\
\hline $\mathrm{T}_{1}$ & $0.80 \mathrm{~g} \pm 0.013$ & $0.11^{\mathrm{e}} \pm 0.009$ & $0.55^{\mathrm{f}} \pm 0.017$ & $0.09^{f} \pm 0.006$ & $0.10^{g} \pm 0.006$ \\
\hline $\mathrm{T}_{2}$ & $1.30^{\mathrm{f}} \pm 0.013$ & $0.18^{\mathrm{d}} \pm 0.009$ & $1.04^{\mathrm{e}} \pm 0.011$ & $0.11^{\mathrm{f}} \pm 0.009$ & $0.13^{g} \pm 0.008$ \\
\hline $\mathrm{T}_{3}$ & $2.25^{b c} \pm 0.016$ & $0.26^{b c} \pm 0.008$ & $2.37^{\mathrm{a}} \pm 0.006$ & $0.54^{\mathrm{cd}} \pm 0.009$ & $0.33^{b} \pm 0.006$ \\
\hline $\mathrm{T}_{4}$ & $2.16^{\mathrm{cd}} \pm 0.022$ & $0.28^{\mathrm{ab}} \pm 0.006$ & $2.26^{\mathrm{d}} \pm 0.006$ & $0.50^{\mathrm{de}} \pm 0.005$ & $0.26 \mathrm{~d}^{\text {ef }} \pm 0.006$ \\
\hline $\mathrm{T}_{5}$ & $2.30^{b} \pm 0.022$ & $0.24^{c} \pm 0.008$ & $2.28^{\mathrm{cd}} \pm 0.009$ & $0.58^{b c} \pm 0.009$ & $0.28^{\text {de }} \pm 0.003$ \\
\hline $\mathrm{T}_{6}$ & $2.41^{\mathrm{a}} \pm 0.021$ & $0.28^{\mathrm{ab}} \pm 0.006$ & $2.25^{\mathrm{d}} \pm 0.006$ & $0.63^{a} \pm 0.010$ & $0.23^{f} \pm 0.011$ \\
\hline $\mathrm{T}_{7}$ & $2.25^{b c} \pm 0.021$ & $0.23^{c} \pm 0.008$ & $2.31^{b c} \pm 0.008$ & $0.60^{\mathrm{ab}} \pm 0.009$ & $0.38^{\mathrm{a}} \pm 0.004$ \\
\hline $\mathrm{T}_{8}$ & $2.14^{\mathrm{d}} \pm 0.013$ & $0.24^{\mathrm{c}} \pm 0.006$ & $2.26^{\mathrm{d}} \pm 0.004$ & $0.54^{\mathrm{cd}} \pm 0.009$ & $0.25^{\text {ef }} \pm 0.006$ \\
\hline $\mathrm{T}_{9}$ & $2.02^{\mathrm{e}} \pm 0.028$ & $0.29^{\mathrm{ab}} \pm 0.006$ & $2.33^{\mathrm{ab}} \pm 0.004$ & $0.60^{\mathrm{ab}} \pm 0.013$ & $0.29^{\mathrm{cd}} \pm 0.012$ \\
\hline $\mathrm{T}_{10}$ & $2.18^{\mathrm{cd}} \pm 0.020$ & $0.31^{\mathrm{a}} \pm 0.008$ & $2.37^{a} \pm 0.013$ & $0.54^{\mathrm{cd}} \pm 0.006$ & $0.32^{b c} \pm 0.006$ \\
\hline $\mathrm{T}_{11}$ & $2.30^{b} \pm 0.019$ & $0.28^{\mathrm{ab}} \pm 0.005$ & $2.33^{\mathrm{ab}} \pm 0.005$ & $0.48^{\mathrm{e}} \pm 0.013$ & $0.24^{\mathrm{f}} \pm 0.009$ \\
\hline$p$-value & $<0.0001$ & $<0.0001$ & $<0.0001$ & $<0.0001$ & $<0.0001$ \\
\hline
\end{tabular}

Means within the same column followed by the same letter are not significantly different at $p \leq 0.05$; (Tukey's HSD test). The column represents the mean values \pm standard error. $\mathrm{T}_{1}=$ No treatments and fertilizer $(\mathrm{Control}), \mathrm{T}_{2}=$ Recommended rate of NPK $\left(\mathrm{t}\right.$ ha $\left.{ }^{-1}\right)$, $\mathrm{T}_{3}=100 \%$ dolomitic limestone $+10 \mathrm{t} \mathrm{ha}^{-1}$ rice husk biochar $+100 \% \mathrm{TSP}, \mathrm{T}_{4}=100 \%$ dolomitic limestone $+10 \mathrm{tha}^{-1}$ rice husk biochar $+75 \%$ TSP, $\mathrm{T}_{5}=100 \%$ dolomitic limestone $+10 \mathrm{tha}^{-1}$ rice husk biochar $+50 \%$ TSP, $\mathrm{T}_{6}=75 \%$ dolomitic limestone $+10 \mathrm{t} \mathrm{ha}^{-1}$ rice husk biochar $+100 \%$ TSP, $\mathrm{T}_{7}=75 \%$ dolomitic limestone $+10 \mathrm{tha}^{-1}$ rice husk biochar $+75 \%$ TSP, $\mathrm{T}_{8}=75 \%$ dolomitic limestone $+10 \mathrm{tha}{ }^{-1}$ rice husk biochar $+50 \%$ TSP, $\mathrm{T}_{9}=75 \%$ dolomitic limestone $+15 \mathrm{tha}^{-1}$ rice husk biochar $+100 \%$ TSP, $\mathrm{T}_{10}=75 \%$ dolomitic limestone $+15 \mathrm{t}$ ha ${ }^{-1}$ rice husk biochar $+75 \%$ TSP, $\mathrm{T}_{11}=75 \%$ dolomitic limestone $+15 \mathrm{t} \mathrm{ha}^{-1}$ rice husk biochar $+50 \%$ TSP.

Table 8. Effect of treatments on changes in plant nutrient uptake.

\begin{tabular}{|c|c|c|c|c|c|}
\hline Treatment & $\frac{\mathrm{N}}{\left(\mathrm{mg} \text { Plant }^{-1}\right)}$ & $\begin{array}{c}\text { P } \\
\left(\mathrm{mg} \mathrm{Plant}^{-1}\right)\end{array}$ & $\begin{array}{c}\mathrm{K} \\
\left(\mathrm{mg} \mathrm{Plant}^{-1}\right)\end{array}$ & $\begin{array}{c}\text { Ca } \\
\left(\mathrm{mg} \mathrm{Plant}^{-1}\right)\end{array}$ & $\begin{array}{c}\text { Mg } \\
\left(\mathrm{mg} \mathrm{Plant}^{-1}\right)\end{array}$ \\
\hline $\mathrm{T}_{1}$ & $178.56 \mathrm{~g} \pm 2.88$ & $24.00^{\mathrm{e}} \pm 1.91$ & $123.32^{\mathrm{i}} \pm 3.91$ & $20.83 \mathrm{~g} \pm 1.08$ & $21.21^{\mathrm{f}} \pm 1.44$ \\
\hline $\mathrm{T}_{2}$ & $511.81^{\mathrm{f}} \pm 5.08$ & $71.42^{\mathrm{d}} \pm 3.43$ & $408.46^{h} \pm 4.36$ & $44.09 \mathrm{~g} \pm 3.37$ & $50.20^{f} \pm 2.95$ \\
\hline $\mathrm{T}_{3}$ & $1886.02^{b} \pm 13.06$ & $216.33^{b} \pm 6.30$ & $1986.84^{\mathrm{a}} \pm 5.42$ & $451.55^{c} \pm 7.18$ & $273.03^{\mathrm{a}} \pm 5.42$ \\
\hline $\mathrm{T}_{4}$ & $1711.37^{\mathrm{d}} \pm 17.42$ & $217.32^{b} \pm 5.19$ & $1786.64^{\mathrm{f}} \pm 5.11$ & $394.17^{\text {ef }} \pm 3.79$ & $229.77^{b c} \pm 10.23$ \\
\hline $\mathrm{T}_{5}$ & $1840.69^{b c} \pm 17.59$ & $190.08^{c} \pm 6.00$ & 1826.69 de \pm 6.83 & $462.17^{b c} \pm 6.83$ & $221.43^{\mathrm{cd}} \pm 3.14$ \\
\hline $\mathrm{T}_{6}$ & $2027.66^{\mathrm{a}} \pm 17.72$ & $231.86^{\mathrm{ab}} \pm 5.44$ & $1892.76^{c} \pm 5.44$ & $526.94^{\mathrm{a}} \pm 8.78$ & $191.81^{\text {de }} \pm 9.35$ \\
\hline $\mathrm{T}_{7}$ & $1844.12^{b c} \pm 17.49$ & $186.26^{c} \pm 6.14$ & $1889.15^{c} \pm 6.14$ & $491.22^{b} \pm 7.47$ & $272.22^{\mathrm{a}} \pm 6.99$ \\
\hline $\mathrm{T}_{8}$ & 1615.59 e \pm 9.40 & $177.12^{\mathrm{c}} \pm 4.87$ & $1703.36 \mathrm{~g} \pm 3.08$ & $408.88^{\text {de }} \pm 6.44$ & $186.69^{\mathrm{e}} \pm 4.85$ \\
\hline $\mathrm{T}_{9}$ & $1590.04^{\mathrm{e}} \pm 22.43$ & $229.33^{\mathrm{ab}} \pm 4.29$ & $1838.63^{\mathrm{d}} \pm 3.22$ & $473.46^{b c} \pm 10.19$ & $224.18^{c} \pm 9.90$ \\
\hline $\mathrm{T}_{10}$ & $1781.36^{\mathrm{cd}} \pm 16.45$ & $250.98^{a} \pm 6.12$ & $1934.39^{b} \pm 10.54$ & $440.75^{\mathrm{cd}} \pm 4.71$ & $257.10^{\mathrm{ab}} \pm 5.27$ \\
\hline $\mathrm{T}_{11}$ & $1777.48^{\mathrm{cd}} \pm 15.00$ & $219.28^{b} \pm 3.65$ & $1802.65^{\text {ef }} \pm 3.71$ & $371.77^{\mathrm{f}} \pm 10.00$ & $176.20^{\mathrm{e}} \pm 6.61$ \\
\hline$p$-value & $<0.0001$ & $<0.0001$ & $<0.0001$ & $<0.0001$ & $<0.0001$ \\
\hline
\end{tabular}

Means within the same column followed by the same letter are not significantly different at $p \leq 0.05$; (Tukey's HSD test). The column represents the mean values \pm standard error. $\mathrm{T}_{1}=$ No treatments and fertilizer (Control), $\mathrm{T}_{2}=$ Recommended rate of NPK $\left(\mathrm{t}\right.$ ha $\left.{ }^{-1}\right)$, $\mathrm{T}_{3}=100 \%$ dolomitic limestone $+10 \mathrm{t} \mathrm{ha}^{-1}$ rice husk biochar $+100 \% \mathrm{TSP}, \mathrm{T}_{4}=100 \%$ dolomitic limestone $+10 \mathrm{tha}^{-1}$ rice husk biochar $+75 \%$ TSP, $\mathrm{T}_{5}=100 \%$ dolomitic limestone $+10 \mathrm{t} \mathrm{ha}^{-1}$ rice husk biochar $+50 \%$ TSP, $\mathrm{T}_{6}=75 \%$ dolomitic limestone $+10 \mathrm{t} \mathrm{ha}^{-1}$ rice husk biochar $+100 \%$ TSP, $\mathrm{T}_{7}=75 \%$ dolomitic limestone $+10 \mathrm{t} \mathrm{ha}^{-1}$ rice husk biochar $+75 \% \mathrm{TSP}, \mathrm{T}_{8}=75 \%$ dolomitic limestone $+10 \mathrm{tha} \mathrm{t}^{-1}$ rice husk biochar $+50 \%$ TSP, $\mathrm{T}_{9}=75 \%$ dolomitic limestone $+15 \mathrm{tha}^{-1}$ rice husk biochar $+100 \%$ TSP, $\mathrm{T}_{10}=75 \%$ dolomitic limestone $+15 \mathrm{tha}{ }^{-1}$ rice husk biochar $+75 \%$ TSP, $\mathrm{T}_{11}=75 \%$ dolomitic limestone $+15 \mathrm{t} \mathrm{ha}^{-1}$ rice husk biochar $+50 \%$ TSP. 


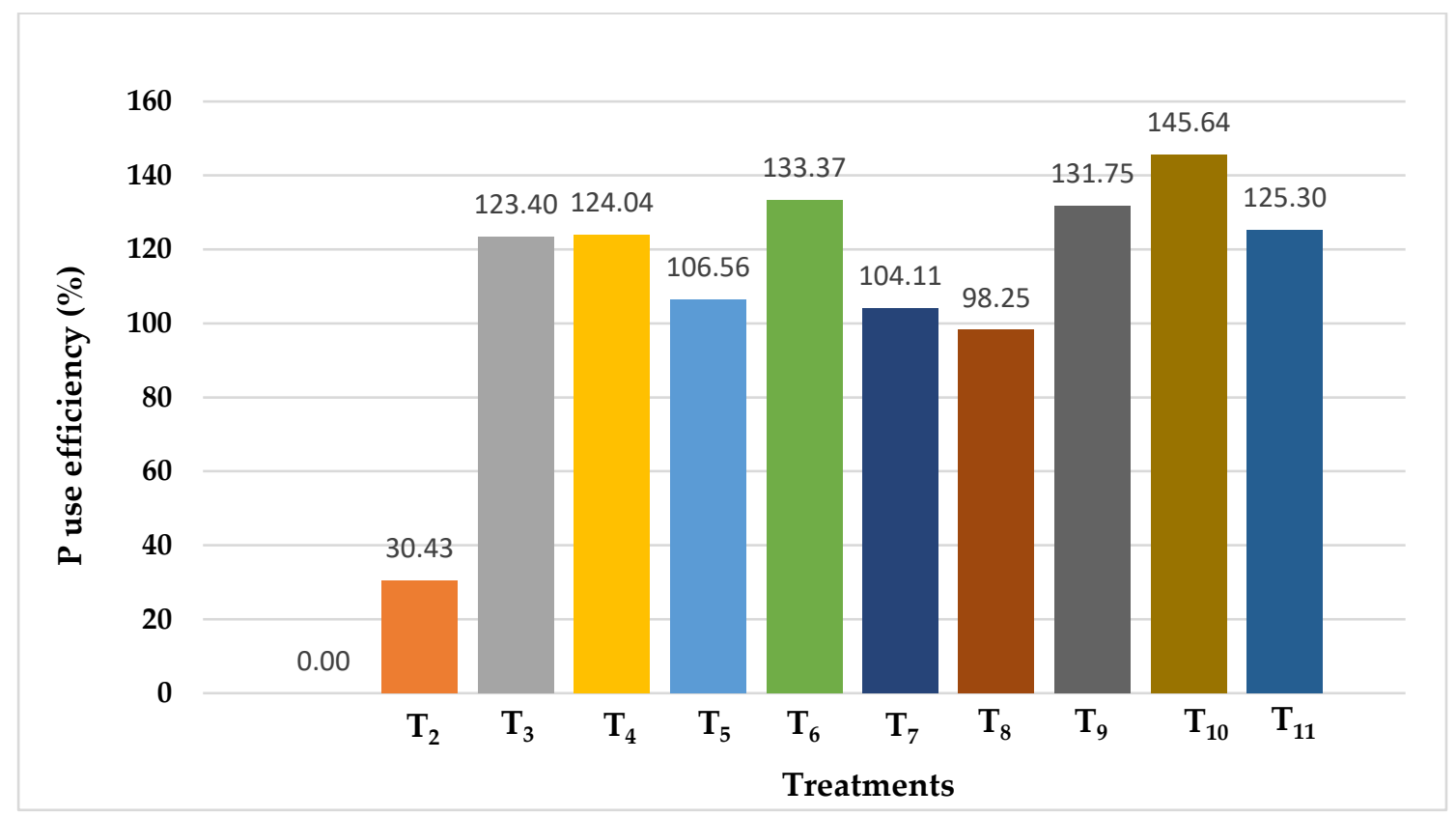

Figure 2. Effect of treatments on phosphorus use efficiency. $\mathrm{T}_{1}=$ No treatments and fertilizer (Control), $\mathrm{T}_{2}=$ Recommended rate of NPK $\left(\mathrm{tha}^{-1}\right), \mathrm{T}_{3}=100 \%$ dolomitic limestone $+10 \mathrm{t} \mathrm{ha}^{-1}$ rice husk biochar $+100 \% \mathrm{TSP}, \mathrm{T}_{4}=100 \%$ dolomitic limestone $+10 \mathrm{t} \mathrm{ha}^{-1}$ rice husk biochar $+75 \%$ TSP, $\mathrm{T}_{5}=100 \%$ dolomitic limestone $+10 \mathrm{tha}{ }^{-1}$ rice husk biochar $+50 \% \mathrm{TSP}, \mathrm{T}_{6}=75 \%$ dolomitic limestone $+10 \mathrm{tha}^{-1}$ rice husk biochar $+100 \%$ TSP, $\mathrm{T}_{7}=75 \%$ dolomitic limestone $+10 \mathrm{t} \mathrm{ha}^{-1}$ rice husk biochar + $75 \% \mathrm{TSP}, \mathrm{T}_{8}=75 \%$ dolomitic limestone $+10 \mathrm{tha}^{-1}$ rice husk biochar $+50 \% \mathrm{TSP}, \mathrm{T}_{9}=75 \%$ dolomitic limestone $+15 \mathrm{t}$ ha ${ }^{-1}$ rice husk biochar $+100 \% \mathrm{TSP}, \mathrm{T}_{10}=75 \%$ dolomitic limestone $+15 \mathrm{t} \mathrm{ha}^{-1}$ rice husk biochar $+75 \% \mathrm{TSP}, \mathrm{T}_{11}=75 \%$ dolomitic limestone $+15 \mathrm{t} \mathrm{ha}^{-1}$ rice husk biochar $+50 \%$ TSP.

\subsection{Effect of Treatments on Changes in Soil $\mathrm{CO}_{2}$ Emission}

Results for the measured $\mathrm{CO}_{2}$ emissions from the treatments are presented in Figure 3, and their cumulative emission dynamic is shown in Figure 4. In this study, $\mathrm{CO}_{2}$ emissions were relatively higher with the co-application of biochar and lime rather than unamended soil. The emission of $\mathrm{CO}_{2}$ was highest on day 5 compared to all other days, from all the treatments except $\mathrm{T}_{1}$ and $\mathrm{T}_{2}$. Among the biochar and lime amended treatments, $\mathrm{T}_{8}(75 \%$ lime $+10 \mathrm{tha}^{-1} \mathrm{RHB}+50 \%$ TSP) emitted the lower $\mathrm{CO}_{2}$ gas, and its cumulative emission was $151.04 \mu \mathrm{mol} \mathrm{CO} \mathrm{Cm}^{-2}$; even though there was no statistically significant difference for $\mathrm{T}_{6}, \mathrm{~T}_{7}, \mathrm{~T}_{8}, \mathrm{~T}_{9}$, and $\mathrm{T}_{10}$. The highest cumulative $\mathrm{CO}_{2}$ flux $\left(160.70 \mu \mathrm{mol} \mathrm{CO}_{2} \mathrm{~m}^{-2}\right)$ was observed from $\mathrm{T}_{3}\left(100 \%\right.$ lime $\left.+10 \mathrm{tha}^{-1} \mathrm{RHB}+100 \% \mathrm{TSP}\right)$ followed by $\mathrm{T}_{4}(157.23 \mu \mathrm{mol}$ $\left.\mathrm{CO}_{2} \mathrm{~m}^{-2}\right)$, and $\mathrm{T}_{5}\left(156.87 \mu \mathrm{mol} \mathrm{CO} \mathrm{Cm}^{-2}\right)$, in contrast, the lowest cumulative $\mathrm{CO}_{2}$ flux $\left(62.25 \mu \mathrm{mol} \mathrm{CO} \mathrm{C} \mathrm{m}^{-2}\right)$ was from the control treatment $\left(\mathrm{T}_{1}\right)$. From Figures 3 and 4 , it is clear that the amount of $\mathrm{CO}_{2}$ emission was very close from all the amended treatments, and the more lime rate, the more $\mathrm{CO}_{2}$ gas emission. The cumulative emission of $\mathrm{CO}_{2}$ was significantly increased by $158.15 \%$ from $\mathrm{T}_{3}\left(100 \%\right.$ lime $\left.+10 \mathrm{t} \mathrm{ha}^{-1} \mathrm{RHB}+100 \% \mathrm{TSP}\right)$, whereas, $142.63 \%$ by $\mathrm{T}_{8}\left(75 \%\right.$ lime $\left.+10 \mathrm{tha}^{-1} \mathrm{RHB}+50 \% \mathrm{TSP}\right)$ compared over control treatment. The cumulative $\mathrm{CO}_{2}$ emission was in the order of $\mathrm{T}_{3}>\mathrm{T}_{4}>\mathrm{T}_{5}>\mathrm{T}_{9}>\mathrm{T}_{7}>\mathrm{T}_{6}>$ $\mathrm{T}_{10}>\mathrm{T}_{11}>\mathrm{T}_{8}>\mathrm{T}_{2}>\mathrm{T}_{1}$.

\subsection{Relationship between Plant Parameters, Nutrients Uptake, Soil $p H$, and Nutrients}

Pearson's correlation analysis was performed to evaluate the relationship among the soil nutrients, plant parameters, plant nutrient uptake, and $\mathrm{CO}_{2}$ emission (Table 9). Grain yield was significantly and positively correlated with soil $\mathrm{pH}(\mathrm{r}=0.98)$, Soil available $\mathrm{P}$ $(\mathrm{r}=0.74)$, exchangeable $\mathrm{K}, \mathrm{Ca}, \mathrm{Mg}(\mathrm{r}=0.97,0.98$, and 0.0 .95 , respectively), extractable $\mathrm{Mn}(\mathrm{r}=0.93)$, plant biomass $(\mathrm{r}=0.97)$, root length $(\mathrm{r}=0.91)$. Furthermore, $\mathrm{CO}_{2}$ flux was correlated with soil $\mathrm{pH}(\mathrm{r}=0.99)$, available $\mathrm{P}(\mathrm{r}=0.76)$, exchangeable $\mathrm{K}, \mathrm{Ca}, \mathrm{Mg}(\mathrm{r}=0.99$. 
0.99 , and 0.97 respectively), extractable $\mathrm{Mn}(\mathrm{r}=0.95)$, plant biomass $(\mathrm{r}=0.97)$, root length $(\mathrm{r}=0.90)$ and grain yield $(\mathrm{r}=0.99)$.

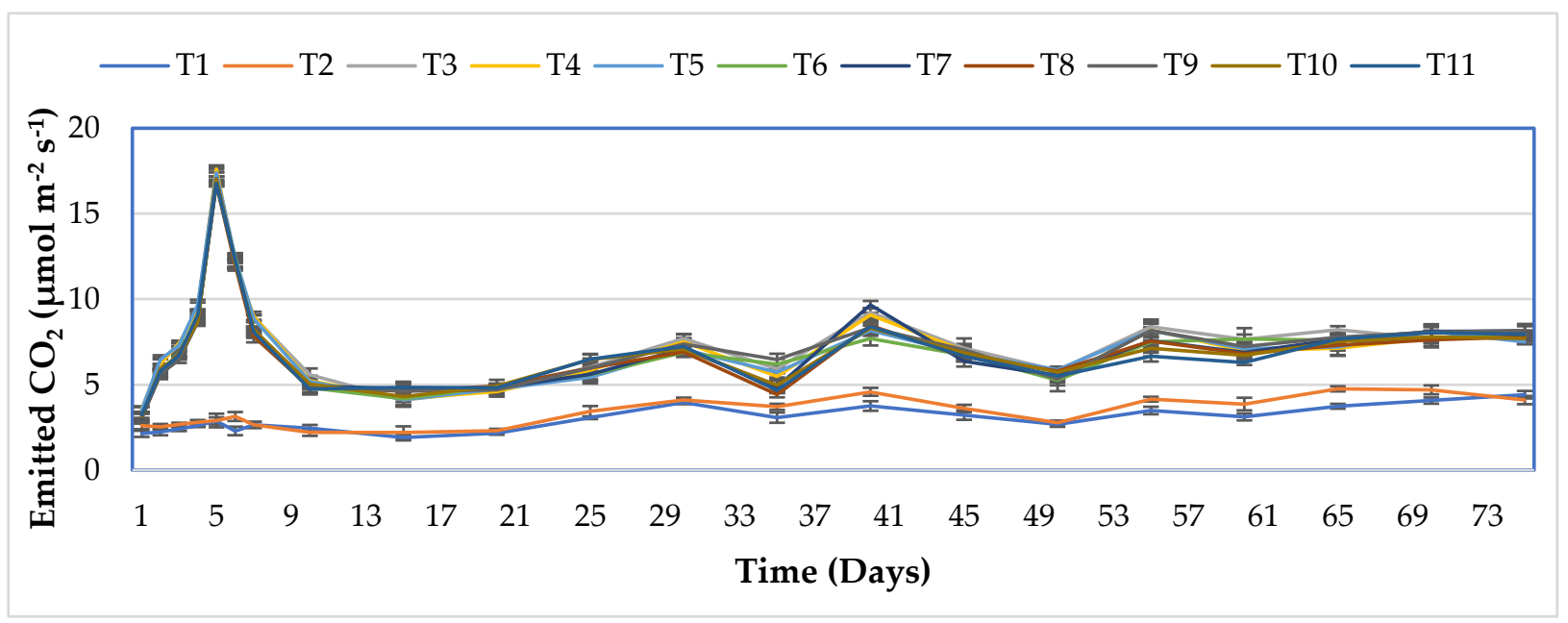

Figure 3. Effect of Treatments on soil $\mathrm{CO}_{2}$ flux emission in a maize field. Bar errors show \pm standard error of four replications. $\mathrm{T}_{1}=$ No treatments and fertilizer (Control), $\mathrm{T}_{2}=\operatorname{Recommended~rate~of~NPK~}\left(\mathrm{t} \mathrm{ha}^{-1}\right), \mathrm{T}_{3}=100 \%$ dolomitic limestone $+10 \mathrm{tha}^{-1}$ rice husk biochar $+100 \% \mathrm{TSP}, \mathrm{T}_{4}=100 \%$ dolomitic limestone $+10 \mathrm{t} \mathrm{ha}{ }^{-1}$ rice husk biochar $+75 \% \mathrm{TSP}$, $\mathrm{T}_{5}=100 \%$ dolomitic limestone $+10 \mathrm{t} \mathrm{ha}^{-1}$ rice husk biochar $+50 \% \mathrm{TSP}, \mathrm{T}_{6}=75 \%$ dolomitic limestone $+10 \mathrm{t} \mathrm{ha}^{-1}$ rice husk biochar $+100 \%$ TSP, $\mathrm{T}_{7}=75 \%$ dolomitic limestone $+10 \mathrm{t} \mathrm{ha}^{-1}$ rice husk biochar $+75 \% \mathrm{TS} \mathrm{P}, \mathrm{T}_{8}=75 \%$ dolomitic limestone + $10 \mathrm{t} \mathrm{ha}^{-1}$ rice husk biochar $+50 \% \mathrm{TSP}, \mathrm{T}_{9}=75 \%$ dolomitic limestone $+15 \mathrm{t} \mathrm{ha}^{-1}$ rice husk biochar $+100 \% \mathrm{TSP}, \mathrm{T}_{10}=75 \%$ dolomitic limestone $+15 \mathrm{t} \mathrm{ha}^{-1}$ rice husk biochar $+75 \%$ TSP, $\mathrm{T}_{11}=75 \%$ dolomitic limestone $+15 \mathrm{t} \mathrm{ha}^{-1}$ rice husk biochar + $50 \%$ TSP.

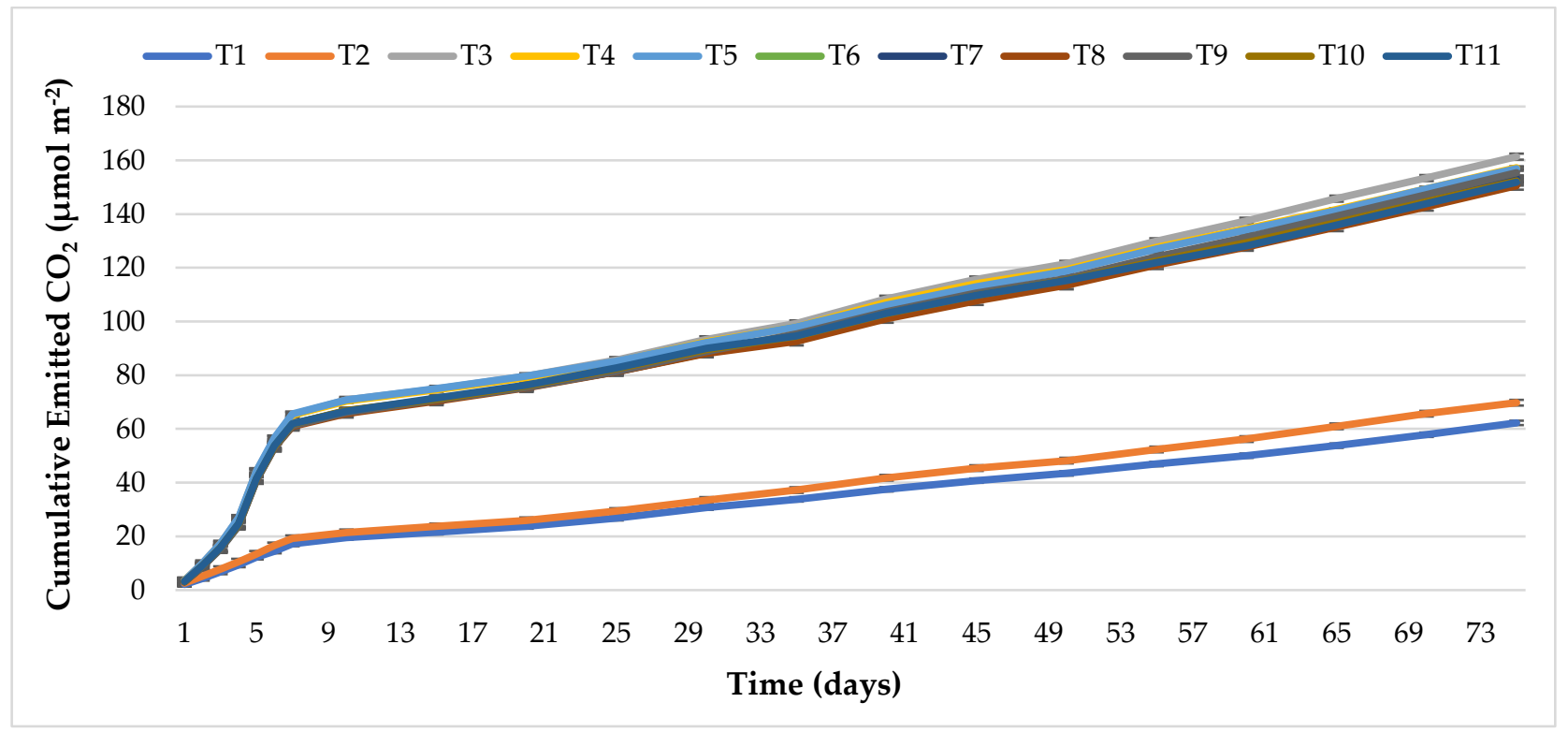

Figure 4. Effect of Treatments on cumulative soil $\mathrm{CO}_{2}$ flux emission. Bar errors show \pm standard error of four replications. $\mathrm{T}_{1}=$ No treatments and fertilizer (Control), $\mathrm{T}_{2}=$ Recommended rate of NPK $\left(\mathrm{t} \mathrm{ha}{ }^{-1}\right), \mathrm{T}_{3}=100 \%$ dolomitic limestone + $10 \mathrm{t} \mathrm{ha}^{-1}$ rice husk biochar $+100 \% \mathrm{TSP}, \mathrm{T}_{4}=100 \%$ dolomitic limestone $+10 \mathrm{tha}^{-1}$ rice husk biochar $+75 \% \mathrm{TSP}, \mathrm{T}_{5}=100 \%$ dolomitic limestone $+10 \mathrm{tha}^{-1}$ rice husk biochar $+50 \% \mathrm{TSP}, \mathrm{T}_{6}=75 \%$ dolomitic limestone $+10 \mathrm{tha}^{-1}$ rice husk biochar + $100 \% \mathrm{TSP}, \mathrm{T}_{7}=75 \%$ dolomitic limestone $+10 \mathrm{t} \mathrm{ha}^{-1}$ rice husk biochar $+75 \% \mathrm{TSP}, \mathrm{T}_{8}=75 \%$ dolomitic limestone $+10 \mathrm{t}$ ha ${ }^{-1}$ rice husk biochar $+50 \% \mathrm{TSP}, \mathrm{T}_{9}=75 \%$ dolomitic limestone $+15 \mathrm{t} \mathrm{ha}^{-1}$ rice husk biochar $+100 \% \mathrm{TSP}, \mathrm{T}_{10}=75 \%$ dolomitic limestone $+15 \mathrm{t} \mathrm{ha}^{-1}$ rice husk biochar $+75 \%$ TSP, $\mathrm{T}_{11}=75 \%$ dolomitic limestone $+15 \mathrm{tha}^{-1}$ rice husk biochar $+50 \% \mathrm{TSP}$. 
Table 9. The correlation coefficient among maize plant parameters, nutrients uptake, soil $\mathrm{pH}$, and nutrients (all relations were significant).

\begin{tabular}{|c|c|c|c|c|c|c|c|c|c|c|c|c|c|c|c|c|c|c|}
\hline Correlation & $\mathrm{pH}$ & Av. $\mathbf{P}$ & $\begin{array}{l}\text { Exch. } \\
\text { K }\end{array}$ & $\begin{array}{l}\text { Exch. } \\
\text { Ca }\end{array}$ & $\begin{array}{l}\text { Exch. } \\
\text { Mg }\end{array}$ & $\begin{array}{l}\text { Exch. } \\
\text { Al }\end{array}$ & $\begin{array}{l}\text { Exct. } \\
\text { Fe }\end{array}$ & $\begin{array}{l}\text { Exct. } \\
\text { Mn }\end{array}$ & $\begin{array}{c}\text { Exch. } \\
\text { Acidity }\end{array}$ & $\begin{array}{c}\text { Plant } \\
\text { Biomass }\end{array}$ & $\begin{array}{c}\text { Root } \\
\text { Length }\end{array}$ & $\begin{array}{l}\text { Grain } \\
\text { Yield }\end{array}$ & $\begin{array}{c}\mathrm{N} \\
\text { Uptake }\end{array}$ & $\begin{array}{c}\mathbf{P} \\
\text { Uptake }\end{array}$ & $\begin{array}{c}\text { K } \\
\text { Uptake }\end{array}$ & $\begin{array}{c}\text { Ca } \\
\text { Uptake }\end{array}$ & $\begin{array}{c}\mathrm{Mg} \\
\text { Uptake }\end{array}$ & $\begin{array}{c}\mathrm{CO}_{2} \\
\text { Emission }\end{array}$ \\
\hline $\mathrm{pH}$ & 1.00 & & & & & & & & & & & & & & & & & \\
\hline Av. P & 0.73 & $1.00 *$ & & & & & & & & & & & & & & & & \\
\hline Exch. K & 0.97 * & 0.77 * & $1.00 *$ & & & & & & & & & & & & & & & \\
\hline Exch. Ca & $0.98 *$ & $0.77 *$ & $0.99 *$ & $1.00 *$ & & & & & & & & & & & & & & \\
\hline Exch. $\mathrm{Mg}$ & $0.95 *$ & $0.86 *$ & $0.97 *$ & $0.97 *$ & $1.00 *$ & & & & & & & & & & & & & \\
\hline Exch Al & $-0.99 *$ & $-0.72 *$ & $-0.98^{*}$ & $-0.98^{*}$ & -0.94 * & $1.00 *$ & & & & & & & & & & & & \\
\hline Exct. Fe & $-0.92 *$ & $-0.82 *$ & $-0.94 *$ & $-0.95^{*}$ & $-0.92 *$ & $0.93 *$ & $1.00 *$ & & & & & & & & & & & \\
\hline Exct. Mn & $0.93 *$ & $0.90 *$ & $0.95 *$ & $0.96 *$ & $0.99 *$ & $-0.92 *$ & $-0.93 *$ & $1.00 *$ & & & & & & & & & & \\
\hline Exch. acidity & $-0.98 *$ & $-0.72 *$ & $-0.97^{*}$ & $-0.97^{*}$ & -0.94 * & $0.98^{*}$ & $0.90 *$ & $-0.91 *$ & $1.00 *$ & & & & & & & & & \\
\hline Plant biomass & $0.96 *$ & $0.78 *$ & $0.97 *$ & $0.97 *$ & $0.95 *$ & $-0.96^{*}$ & $-0.92 *$ & $0.93 *$ & $-0.98 *$ & $1.00 *$ & & & & & & & & \\
\hline Root length & $0.91 *$ & $0.63 *$ & $0.91 *$ & $0.90 *$ & $0.84 *$ & $-0.91 *$ & $-0.88^{*}$ & $0.82 *$ & -0.93 * & $0.90 *$ & $1.00 *$ & & & & & & & \\
\hline Grain yield & $0.98 *$ & $0.74 *$ & $0.97 *$ & $0.98 *$ & $0.95 *$ & $-0.98 *$ & $-0.93^{*}$ & $0.93 *$ & $-0.98 *$ & 0.97 * & $0.91 *$ & $1.00 *$ & & & & & & \\
\hline $\mathrm{N}$ uptake & $0.97 *$ & $0.75^{*}$ & $0.96^{*}$ & $0.96 *$ & $0.94 *$ & $-0.97 *$ & $-0.92 *$ & $0.92 *$ & $-0.98 *$ & 0.97 * & $0.92 *$ & 0.98 * & $1.00 *$ & & & & & \\
\hline P uptake & $0.90 *$ & $0.81 *$ & $0.94 *$ & $0.95 *$ & $0.96 *$ & $-0.90 *$ & $-0.91 *$ & $0.94 *$ & $-0.92 *$ & $0.93 *$ & 0.82 & $0.94 *$ & $0.93 *$ & $1.00 *$ & & & & \\
\hline K uptake & 0.98 * & $0.79 *$ & $0.98 *$ & $0.99 *$ & $0.97 *$ & $-0.98 *$ & $-0.94 *$ & $0.96 *$ & $-0.98 *$ & $0.98 *$ & $0.91 *$ & $0.99 *$ & $0.99 *$ & $0.96 *$ & $1.00 *$ & & & \\
\hline Ca uptake & 0.96 * & $0.81 *$ & $0.97 *$ & $0.96 *$ & $0.94 *$ & $-0.96^{*}$ & $-0.95 *$ & $0.93 *$ & $-0.95 *$ & 0.96 * & $0.88^{*}$ & $0.96 *$ & 0.97 * & $0.91 *$ & $0.97 *$ & $1.00 *$ & & \\
\hline Mg uptake & $0.92 *$ & $0.78^{*}$ & $0.89 *$ & $0.94 *$ & $0.92 *$ & $-0.91 *$ & $-0.90 *$ & $0.91 *$ & -0.91 * & 0.91 * & $0.83 *$ & $0.91 *$ & $0.91 *$ & 0.87 * & 0.94 * & $0.91 *$ & $1.00 *$ & \\
\hline $\mathrm{CO}_{2}$ emission & $0.99 *$ & $0.76^{*}$ & $0.99 *$ & $0.99 *$ & $0.97^{*}$ & -0.99 * & $-0.94^{*}$ & $0.95 *$ & $-0.98 *$ & $0.97 *$ & $0.90 *$ & $0.99 *$ & $0.97 *$ & $0.93 *$ & 0.99 * & $0.96^{*}$ & $0.92 *$ & 1.00 * \\
\hline
\end{tabular}

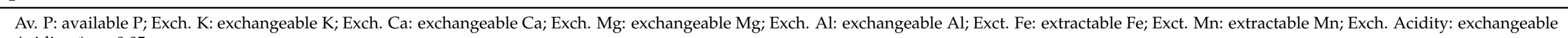
Acidity; ${ }^{*}<0.05$. 


\section{Discussion}

\subsection{Impact of Treatments on Changes in Nutrients of the Post-Harvest Soil}

In this study, the soil $\mathrm{pH}$, at the end of the study, significantly increased due to different soil amendments. Among the treatment combinations, the $\mathrm{pH}$ was relatively higher, except in treatment $\mathrm{T}_{4}$, when $100 \%$ lime was applied, than all other treatments. When $75 \%$ of the recommended rate of biochar was applied along with different rates of biochar, soil $\mathrm{pH}$ was higher when biochar was applied at higher application rates. These results suggest that biochar buffered soil $\mathrm{pH}$ possibly through basic cation additions and $\mathrm{H}^{+}$consumption on its negative functional groups (Table 2). Biochar mediating an increase in soil $\mathrm{pH}$ has been reported by Abdulrahman et al. [54], while organic amendments mediating $\left(\mathrm{H}^{+}\right)$exchange has also been reported [55]. Our result was in line with the study by Ch'ng et al. [56], where they reported an increase of 0.99 units of soil $\mathrm{pH}$ after applying chicken litter biochar.

Soil available $\mathrm{P}$ significantly increased by using the different treatments compared to the unamended soil. The largest increment was observed in the $\mathrm{T}_{9}$, i.e., when biochar was applied at $15 \mathrm{t} \mathrm{ha}^{-1}$ along with $100 \%$ recommended rate of $\mathrm{P}$ and $75 \%$ of the lime requirement. Moreover, the available P was relatively larger when the $100 \% \mathrm{P}$ was applied irrespective of biochar and lime addition than other $\mathrm{P}$ treatments. Under $75 \% \mathrm{P}$ addition, biochar $\left(15 \mathrm{t} \mathrm{ha}^{-1}\right)$ along with $75 \%$ lime performed equally with $\mathrm{T}_{4}(100 \%$ lime, and $10 \mathrm{t}$ biochar ha ${ }^{-1}$ ), suggesting a clear biochar effect. This is plausible since biochar amendment significantly increased soil $\mathrm{pH}$ that might have increased the bioavailability of P. Our presumption is supported by a significant correlation between soil $\mathrm{pH}$ and available soil P (Table 9 and Figure 5) [57]. Besides, freshly prepared biochars with large surface areas perform as an adsorbent of phosphate and help to increase the $\mathrm{P}$ bioavailability of soil [31]. Our result was similar to the findings of Panhwar et al. [58]. By applying rice husk biochar and bio-fertilizer, they revealed $99.82 \%$ increased soil available P.

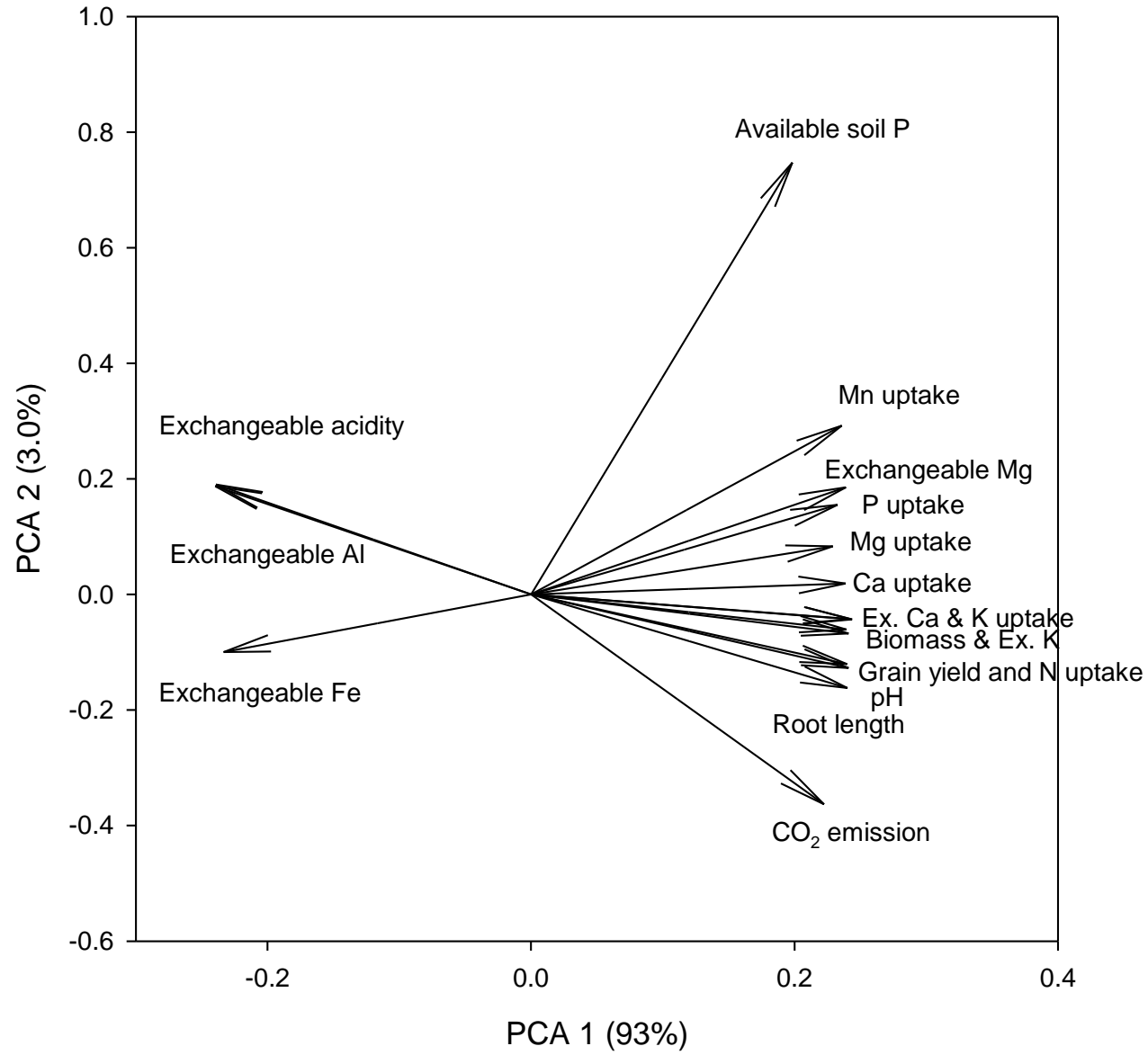

Figure 5. Principal component analysis of different variables. 
A significant increase in base cations $\left(\mathrm{Ca}^{2+}, \mathrm{Mg}^{2+}, \mathrm{K}^{+}\right)$was recorded after applying biochar and lime treatments compared to unamended soil. Our result was well supported by Maru et al. [23], where they found increased exchangeable bases by using $5 \mathrm{tha}^{-1}$ chicken litter biochar in tropical acid soil. Soil exchangeable bases are raised by the exchange reaction between the organic amendment's base cations and soil exchangeable $\mathrm{Al}^{3+}$ and $\mathrm{H}^{+}$. Apart from this, the lime application is another source of soil exchangeable $\mathrm{Ca}$ and $\mathrm{Mg}$. Therefore, these treatments ameliorate soil acidity and provide nutrients in acid soil [59].

All the treatments except $T_{1}$ and $T_{2}$ significantly decreased exchangeable acidity, exchangeable $\mathrm{Al}$, and extractable $\mathrm{Fe}$ after biochar and lime were applied compared to unamended soil. Our result revealed that the exchangeable acidity, Al, and Fe concentration reduced by $95.20 \%, 96.84 \%$, and $45.83 \%$, respectively. This reduction is partially related to the increased soil $\mathrm{pH}$ [56]. Soluble $\mathrm{Al}$ and Fe content precipitated as insoluble hydroxides of $\mathrm{Al}$ and $\mathrm{Fe}$ on the surface of the organic amendments due to the increase in soil $\mathrm{pH}$. Thus, $\mathrm{Al}$ and Fe content was reduced [56]. In addition, exchangeable acidity decreased due to the increased soil $\mathrm{pH}$. Besides, exchangeable acidity is reduced by lowering the exchangeable $\mathrm{Al}$ [60]. Our findings align with Kamran et al. [61], noting that exchangeable acidity was reduced by using chicken manure biochar.

\subsection{Impact of Treatments on Plant Growth and Yield Contributing Characters}

There were significant changes in the growth performance of maize plants when grown in the amended soils compared to unamended soil. In terms of yield, all the amended soils performed equally except for $\mathrm{T}_{9}$. These results suggest that biochar application along with reduced lime and P (up to 50\%) can provide a yield similar to their $100 \%$ recommendation. This yield was possibly manifested by a better plant performance in terms of yield contributing characters under these treatments (Figure 5). The first component in the principal component analysis explained most of the variation (93\%) in the variables, while the second component explained only $3 \%$ of the variations. Among the variables analyzed, all variables had positive loading in the first component except exchangeable acidity, exchangeable $\mathrm{Al}$, and $\mathrm{Fe}$. However, the values of the loading were almost similar for all variables estimating at +0.22 and -0.22 . In the second component, the variation in loading was much prominent ranging from -0.4 for $\mathrm{CO}_{2}$ emission to +0.78 for soil available P. Altogether, our PCA analysis showed that grain yield was positively associated with soil $\mathrm{pH}$, root length, plant $\mathrm{N}, \mathrm{P}, \mathrm{Ca}$, and $\mathrm{Mg}$ uptake. In contrast, it was negatively associated with exchangeable acidity, exchangeable Al, and Fe. Specifically, the better performance of maize in terms of yield and yield components was possibly occurred due to an increase in soil $\mathrm{pH}$ that helps to make the plant nutrients available. A greater availability of nutrients including N, P, K, and others promoted plant growth through a larger nutrient uptake. For instance, available $\mathrm{P}$ was higher in these treatments, and there was a significant relationship between grain yield and available P (Figure 6). Similar trends were also observed for other nutrients (Table 9). Moreover, incorporating biochar into the soil might have minimized the leaching loss of nutrients, thereby influencing plants' growth [62]. Similar results have also been reported in the literature. For instance, an increased soil $\mathrm{pH}$ has been shown as the vital growth factor of maize plants $[63,64]$. According to Mehmood et al. [65], reduced exchangeable acidity increased soil $\mathrm{pH}$, and a sufficient amount of nutrients present in the soil due to biochar addition are considered an enhancement of plant growth. Our findings are also in line with Masud et al. [59], where they used poultry litter biochar to increase maize production in acid soil. Co-application of biochar and lime is supportive to nutrient release and also enhancement of nutrient retention to boost the yield of maize [62]. 

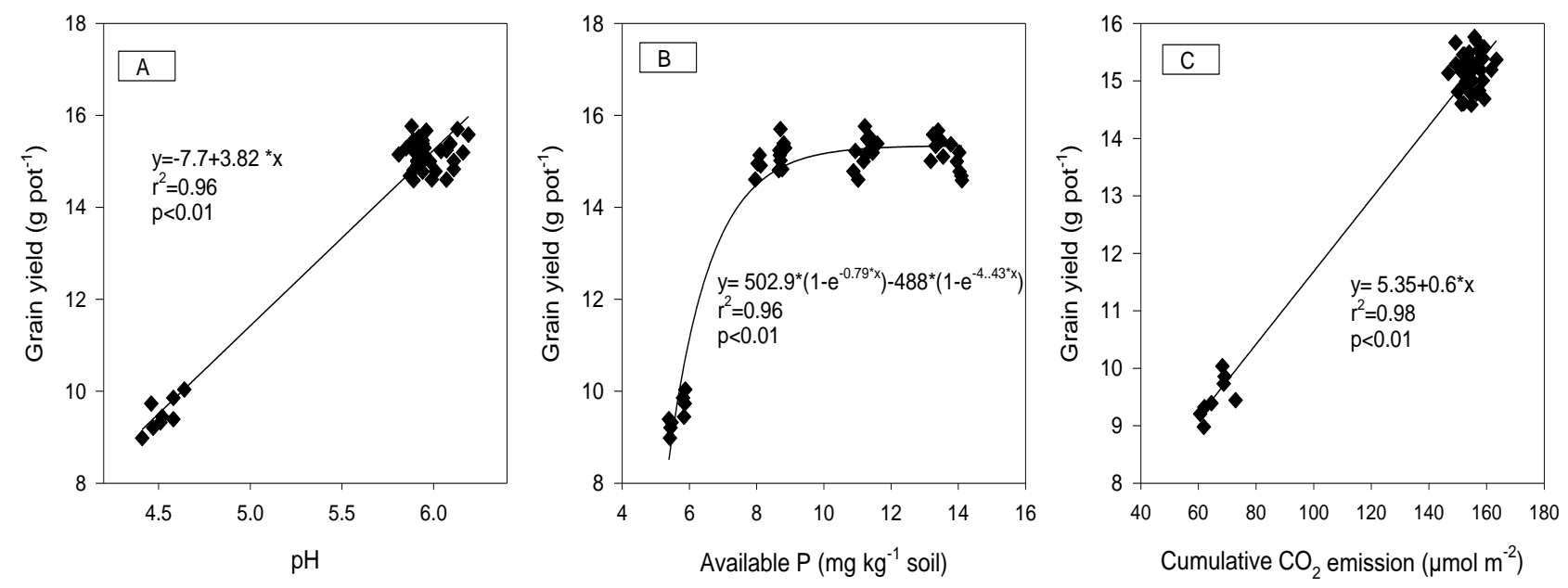

Figure 6. Relationship between soil $\mathrm{pH}$, soil available $\mathrm{P}$, and cumulative $\mathrm{CO}_{2}$ emission against grain yield (A-C), respectively.

\subsection{Impact of Treatments on Plant Nutrient Concentration and Uptake}

Our study showed that nutrient concentration and total uptake were highly significantly increased with the co-application of biochar and lime-treated soil compared to untreated soil. This result is similar to the study by Rabileh et al. [49], where they found increased nutrient uptake treated with biochar and lime. In addition, our study revealed that the application of biochar and lime reduces $\mathrm{Al}$ and Fe content from the soil by increasing soil $\mathrm{pH}$, which helps to reduce the $\mathrm{Al}$ toxicity of roots and resulted in increased nutrient uptake of maize [56]. A meta-analysis conducted by Biederman et al. [66] reported that the amount of $\mathrm{P}$ and $\mathrm{K}$ raised in plants by adding biochar over fertilizer due to increased nutrient availability by increasing retention capacity and soil liming.

\subsection{Impact of Treatments on Changes in Soil $\mathrm{CO}_{2}$ Emission}

The co-application of biochar with lime and different doses of $\mathrm{P}$ fertilizer significantly influenced the emission rate of $\mathrm{CO}_{2}$. In this present study, applied high lime rate contributes to an increase of $158.15 \%$ from T3 (100\% lime $+10 \mathrm{tha}^{-1} \mathrm{RHB}+100 \%$ TSP), while, $142.63 \%$ by $\mathrm{T} 8\left(75 \%\right.$ lime $\left.+10 \mathrm{tha}^{-1} \mathrm{RHB}+50 \% \mathrm{TSP}\right)$ compared to untreated soil $\mathrm{CO}_{2}$ emission. It seems that, for increased $\mathrm{CO}_{2}$ emissions, carbon dissociation is not the only liable fact; whereas, $\mathrm{CO}_{2}$ is emitted due to the prompt activities of microbial communities [67]. In the initial stage of our pot study, the emission of $\mathrm{CO}_{2}$ flux was highest on day 5 from all the amended soil due to the speedy mineralization of soil organic carbon [68]. This result is consistent with the findings of Mosharrof et al. [69]. According to Fidel et al. [70], the daily emission of $\mathrm{CO}_{2}$ was changed because of the variation of density of root and biomass of plants, resulting in the labile soil organic carbon variation.

Generally, the application of biochar into the soil may reduce the emission of $\mathrm{CO}_{2}$ gas from the soil by the labile carbon absorbed on the biochar's surface or into the biochar's pore space [71]. Furthermore, the recalcitrant characteristic of biochar, which helps to decompose slowly and resulted in reduced microbial respiration, is another cause of decreased $\mathrm{CO}_{2}$ flux emission [72]. In contrast, biochar contains volatile organic carbon, contributing to enhancing the soil $\mathrm{CO}_{2}$ emission [73].

\section{Conclusions}

In tropical acidic soil, amending with the combined application of different rates of RHB, dolomitic limestone, and chemical $\mathrm{P}$ fertilizer can enhance nutrients availability to the plant by increasing soil $\mathrm{pH}$, available $\mathrm{P}$, base cations, and reducing exchangeable acidity while reducing the toxicity of $\mathrm{Al}$ and Fe. Compared to control and NPK treatments, combined application of lime and RHB increased P bioavailability with the largest increase in the greater $\mathrm{P}$ application rate. However, the difference between different RHB and lime treatments (i.e., application rates) was minimal. Finally, our results indicate that treatments 
with $75 \%$ lime $+10 t \mathrm{tha}^{-1}$ RHB with different doses of $\mathrm{P}$ fertilizer $\left(\mathrm{T}_{6}, \mathrm{~T}_{7}\right.$ and $\left.\mathrm{T}_{8}\right)$ are recommended, which can significantly increase available $P$ in soil, dry biomass, and yield of maize, instead of $100 \%$ lime or $15 \mathrm{tha}^{-1} \mathrm{RHB}$. This approach will help reduce economic cost, environmental benefits, and gain more profit to farmers; concurrently, reduced soil $\mathrm{CO}_{2}$ gas may reduce the threat of various earth ecosystems. However, further research is needed to evaluate the agronomical values at a framers field scale.

Author Contributions: Conceptualization, M.K.U. and M.F.S.; designed experiments, collected data, conducted statistical analysis M.M., M.K.U. and M.F.S.; validation, M.K.U. and M.F.S.; writingoriginal draft preparation, M.M.; writing-review and editing, M.K.U., S.M. and S.M.S.; visualization, M.M., M.K.U., M.F.S. and A.N.A.H.; supervision, M.K.U. and M.F.S. All authors have read and agreed to the published version of the manuscript.

Funding: This paper was supported by Universiti Putra Malaysia (Vote No. 6282512-10201), Fundamental Research Grant Scheme (FRGS 1/2020/WAB04/ Vote no 5540389) and National Agricultural Technology Programme (NATP): Phase- II Project, Bangladesh Agricultural Research Council.

Institutional Review Board Statement: Not applicable.

Informed Consent Statement: Not applicable.

Data Availability Statement: Not applicable.

Acknowledgments: Authors are grateful to the National Agricultural Technology Program-Phase-II Bangladesh Agricultural Research Council for the financial support and the Universiti Putra Malaysia, Selangor Darul Ehsan, Malaysia, for the research facilities.

Conflicts of Interest: The authors declare no conflict of interest.

\section{References}

1. Zaidun, S.W.; Jalloh, M.B.; Awang, A.; Sam, L.M.; Besar, N.A.; Musta, B.; Ahmed, O.H.; Omar, L. Biochar and clinoptilolite zeolite on selected chemical properties of soil cultivated with maize (Zea mays L.). Eurasian J. Soil Sci. 2019, 8, 1-10. [CrossRef]

2. Agegnehu, G.; Srivastava, A.K.; Bird, M.I. The role of biochar and biochar-compost in improving soil quality and crop performance: A review. Appl. Soil Ecol. 2017, 119, 156-170. [CrossRef]

3. Hasbullah, N.A.; Ahmed, O.H.; Majid, N.M.A. Effects of Amending Phosphatic Fertilizers with Clinoptilolite Zeolite on Phosphorus Availability and Its Fractionation in an Acid Soil. Appl. Sci. 2020, 10, 3162. [CrossRef]

4. Kalkhoran, S.S.; Pannell, D.J.; Thamo, T.; White, B.; Polyakov, M. Soil acidity, lime application, nitrogen fertility, and greenhouse gas emissions: Optimizing their joint economic management. Agric. Syst. 2019, 176, 102684. [CrossRef]

5. Joris, H.A.W.; Caires, E.F.; Scharr, D.A.; Bini, A.R.; Haliski, A. Liming in the conversion from degraded pastureland to a no-till cropping system in Southern Brazil. Soil Tillage Res. 2016, 162, 68-77. [CrossRef]

6. Tiritan, C.S.; Büll, L.T.; Crusciol, C.A.C.; Carmeis Filho, A.C.A.; Fernandes, D.M.; Nascente, A.S. Tillage system and lime application in a tropical region: Soil chemical fertility and corn yield in succession to degraded pastures. Soil Tillage Res. 2016, 155, 437-447. [CrossRef]

7. Li, G.D.; Conyers, M.K.; Helyar, K.R.; Lisle, C.J.; Poile, G.J.; Cullis, B.R. Long-term surface application of lime ameliorates subsurface soil acidity in the mixed farming zone of south-eastern Australia. Geoderma 2019, 338, 236-246. [CrossRef]

8. Gazey, C.; Davies, S.; Master, R. Soil Acidity: A Guide for WA Farmers and Consultant, 2nd ed.; Bulletin 4858; Department of Agriculture and Food: Perth, Australia, 2014.

9. Wang, M.; Xian-Jun, J. Effects of applying lime and calcium montmorillonite on nitrification dynamics in acidic soil. J. Agric. Resour. Environ. 2017, 34, 47. [CrossRef]

10. Mia, S.; Singh, B.; Dijkstra, F.A. Chemically oxidized biochar increases ammonium-15 N recovery and phosphorus uptake in a grassland. Biol. Fertil. Soils 2019, 55, 577-588. [CrossRef]

11. Neumann, G.; Römheld, V. Rhizosphere Chemistry in Relation to Plant Nutrition. In Marschner's Mineral Nutrition of Higher Plants, 3rd ed.; Academic Press: London/Waltham, UK; San Diego, CA, USA, 2011; pp. 347-368.

12. Wu, S.; Zhang, Y.; Tan, Q.; Sun, X.; Wei, W.; Hu, C. Science of the Total Environment Biochar is superior to lime in improving acidic soil properties and fruit quality of Satsuma mandarin. Sci. Total Environ. 2020, 714, 136722. [CrossRef]

13. Novais, S.V.; Zenero, M.D.O.; Barreto, M.S.C.; Montes, C.R.; Cerri, C.E.P. Phosphorus removal from eutrophic water using modified biochar. Sci. Total Environ. 2018, 63, 825-835. [CrossRef]

14. Oni, B.A.; Oziegbe, O.; Olawole, O.O. Significance of biochar application to the environment and economy. Ann. Agric. Sci. 2020, 64, 222-236. [CrossRef]

15. Kavitha, B.; Reddy, P.V.L.; Kim, B.; Lee, S.S.; Pandey, S.K.; Kim, K. Benefits and limitations of biochar amendment in agricultural soils: A review. J. Environ. Manag. 2018, 227, 146-154. [CrossRef] 
16. Adekiya, A.O.; Agbede, T.M.; Olayanju, A.; Ejue, W.S.; Adekanye, T.A.; Adenusi, T.T.; Ayeni, J.F. Effect of Biochar on Soil Properties, Soil Loss, and Cocoyam Yield on a Tropical Sandy Loam Alfisol. Sci. World J. 2020, 2020. [CrossRef]

17. Tomczyk, A.; Sokołowska, Z.; Boguta, P. Biochar physicochemical properties: Pyrolysis temperature and feedstock kind effects. Rev. Environ. Sci. Biotechnol. 2020, 19, 191-215. [CrossRef]

18. Solaiman, Z.M.; Shafi, M.I.; Beamont, E.; Anawar, H.M. Poultry litter biochar increases mycorrhizal colonisation, soil fertility and cucumber yield in a fertigation system on sandy soil. Agriculture 2020, 10, 480. [CrossRef]

19. Deluca, T.H.; Gundale, M.J.; MacKenzie, M.D.; Jones, D.L. Biochar effects on soil nutrient transformations. In Biochar for Environmental Management: Science, Technology and Implementation, 2nd ed.; Lehmann, J., Joseph, S., Eds.; Earthscan: New York, NY, USA, 2015; pp. 424-425.

20. Gul, S.; Whalen, J.K. Biochemical cycling of nitrogen and phosphorus in biochar-amended soils. Soil Biol. Biochem. 2016, 103, 1-15. [CrossRef]

21. Lehmann, J.; Rillig, M.C.; Thies, J.; Masiello, C.A.; Hockaday, W.C.; Crowley, D. Biochar effects on soil biota-A review. Soil Biol. Biochem. 2011, 43, 1812-1836. [CrossRef]

22. Kahura, M.W.; Hyungi, M.; Min, S.K.; Jeong, G.K. Assessing phosphorus availability in a high pH, biochar amended soil under inorganic and organic fertilization. Ecol. Resilient Infrastruct. 2018, 5, 11-18. [CrossRef]

23. Maru, A.; Haruna, A.O.; Asap, A.; Majid, N.M.A.; Maikol, N.; Jeffary, A.V. Reducing acidity of tropical acid soil to improve phosphorus availability and Zea mays L. Productivity through efficient use of chicken litter biochar and triple superphosphate. Appl. Sci. 2020, 10, 2127. [CrossRef]

24. Mia, S.; Van Groenigen, J.W.; Van de Voorde, T.F.J.; Oram, N.J.; Bezemer, T.M.; Mommer, L.; Jeffery, S. Biochar application rate affects biological nitrogen fixation in red clover conditional on potassium availability. Agric. Ecosyst. Environ. 2014, 191, 83-91. [CrossRef]

25. Hiemstra, T.; Mia, S.; Duhaut, P.B.; Molleman, B. Natural and pyrogenic humic acids at goethite and natural oxide surfaces interacting with phosphate. Environ. Sci. Technol. 2013, 47, 9182-9189. [CrossRef] [PubMed]

26. Mia, S.; Dijkstra, F.A.; Singh, B. Aging induced changes in biochar's functionality and adsorption behavior for phosphate and ammonium. Environ. Sci. Technol. 2017, 51, 8359-8367. [CrossRef]

27. Bolan, N.S.; Adriano, D.; Senesi, N.; Kunhikrishnan, A.; James, T.; McDowell, R. Dissolved organic carbon: Biogeochemistry, dynamics and agro environmental significance in soils. Adv. Agron. 2010, 110, 1-67. [CrossRef]

28. Zimmerman, A.R.; Gao, B.; Ahn, M.Y. Positive and negative carbon mineralization priming effects among a variety of biocharamended soils. Soil Biol. Biochem. 2011, 43, 1169-1179. [CrossRef]

29. Wang, J.; Pan, X.; Liu, Y.; Zhang, X.; Xiong, Z. Effects of biochar amendment in two soils on greenhouse gas emissions and crop production. Plant Soil 2012, 360, 287-298. [CrossRef]

30. Thammasom, N.; Vityakon, P.; Lawongsa, P.; Saenjan, P. Biochar and rice straw have different effects on soil productivity, greenhouse gas emission and carbon sequestration in Northeast Thailand paddy soil. Agric. Nat. Resour. 2016, 50, 192-198 [CrossRef]

31. Zhang, H.Z.; Chen, C.R.; Gray, E.M.; Boyd, S.E.; Yang, H.; Zhang, D.K. Roles of biochar in improving phosphorus availability in soils: A phosphate adsorbent and a source of available phosphorus. Geoderma 2016, 276, 1-6. [CrossRef]

32. Soltangheisi, A.; Rodrigues, M.; Coelho, M.J.A.; Gasperini, A.M.; Sartor, L.R.; Pavinato, P.S. Changes in soil phosphorus lability promoted by phosphate sources and cover crops. Soi Tillage Res. 2018, 179, 20-28. [CrossRef]

33. Pedram, K. Genetic Potential of Selected Sweet Corn Inbred Lines and Analysis of Their Combining Ability Assisted by Microsatellite DNA Markers. Ph.D. Thesis, Universiti Putra Malaysia, Seri Kembangan, Selangor, Malaysia, 2012.

34. Mosharrof, M.; Uddin, M.K.; Jusop, S.; Sulaiman, M.F.; Shamsuzzaman, S.M.; Haque, A.N.A. Changes in Acidic Soil Chemical Properties and Carbon Dioxide Emission Due to Biochar and Lime Treatments. Agriculture 2021, 11, 219. [CrossRef]

35. Bouyoucos, G.J. Hydrometer method improved for making particle size analysis of soils. Agron. J. 1962, 54, 464-465. [CrossRef]

36. Soil Survey Staff. Soil Survey Laboratory Methods Manual; Soil Survey Laboratory Investigations Report; U.S. Department of Agriculture Lincoln, Nebraska: Washington, DC, USA, 2014.

37. Tan, K.H. Soil and plant test. In Soil Sampling, Preparation, and Analysis, 2nd ed.; Tan, K.H., Ed.; Taylor \& Francis Group, CRC Press: Boca Raton, FL, USA, 2005; pp. 98-134.

38. Benton, J.J. Laboratory Guide for Conducting Soil Tests and Plant Analysis; CRC Press: Boca Raton, FL, USA, 2001 ; ISBN 9780429132117.

39. Schollenberger, C.J.; Simon, R.H. Determination of exchange capacity and exchangeable bases in soil-ammonium acetate method. Soil Sci. 1945, 59, 13-24. [CrossRef]

40. Elisa, A.A.; Ninomiya, S.; Shamshuddin, J.; Roslan, I. Alleviating aluminum toxicity in an acid sulfate soil from Peninsular Malaysia by calcium silicate application. Solid Earth 2016, 7, 367-374. [CrossRef]

41. Bray, R.H.; Kurtz, L.T. Determination of total, organic, and available forms of phosphorus in soils. Soil Sci. 1945, 59, 39-46. [CrossRef]

42. Douglas, L.A.; Bremner, J.M. Extraction and colorimetric determination of urea in soils. Soil Sci. Soc. Am. 1970, 34, 859-862. [CrossRef]

43. Ahmedna, M.; Marshall, W.E.; Rao, R.M. Production of granular activated carbon from select agricultural by-products and evaluation of their physical, chemical, and adsorption properties. Bioresour. Technol. 1998, 71, 113-123. [CrossRef] 
44. Song, W.; Guo, M. Quality variations of poultry litter biochar generated at different pyrolysis temperature. J. Anal. Appl. Pyrolysis 2012, 94, 138-145. [CrossRef]

45. Lai, L. Utilization of Rice Straw Biochar and Urea to Mitigate Greenhouse Gases Emission in Sustainable Rice Production. Ph.D. Thesis, Universiti Putra Malaysia, Seri Kembangan, Selangor, Malaysia, 2019.

46. Yuan, Z.; Cao, Q.; Zhang, K.; Ata-Ul-Karim, S.T.; Tian, Y.; Zhu, Y.; Cao, W.; Liu, X. Optimal leaf positions for SPAD meter measurement in rice. Front. Plant Sci. 2016, 7, 719. [CrossRef]

47. Lija, M.; Ahmed, O.H.; Susilawati, K. Maize (Zea mays L.) nutrient use efficiency as affected by formulated fertilizer with Clinoptilolite Zeolite. Emir. J. Food Agric. 2014, 26, 284-292. [CrossRef]

48. Cottenie, A. Soil testing and plant testing as a basis of fertilizer recommendation. FAO Soil Bull. 1980, 38, 70-73.

49. Rabileh, M.A.; Shamshuddin, J.; Panhwar, Q.A.; Rosenani, A.B.; Anuar, A.R. Effects of biochar and/or dolomitic limestone application on the properties of Ultisol cropped to maize under glasshouse conditions. Can. J. Soil Sci. 2015, 95, 37-47. [CrossRef]

50. Snyder, C.S.; Bruulsema, T.W. Nutrient Use Efficiency and Effectiveness in North America: Indices of Agronomic and Environmental Benefit; International Plant Nutrition Institute: Norcross, GA, USA, 2007; p. 4.

51. Iqbal, J.; Hu, R.G.; Feng, M.; Lin, S.; Malghani, S.; Ali, I.M. Microbial biomass, and dissolved organic carbon and nitrogen strongly affect soil respiration in different land uses: A case study at Three Gorges Reservoir Area, South China. Agric. Ecosyst. Environ. 2010, 137, 294-307. [CrossRef]

52. Shen, Y.; Zhu, L.; Cheng, H.; Yue, S.; Li, S. Effects of biochar application on $\mathrm{CO}_{2}$ emissions from a cultivated soil under semiarid climate conditions in Northwest China. Sustainability 2017, 9, 1482. [CrossRef]

53. Ashraf, M.; Waheed, A. Screening of local/exotic accessions of lentil (Lens culinaris Medic.) for salt tolerance at two growth stages. Plant Soil 1990, 128, 167-176. [CrossRef]

54. Abdulrahman, D.K.; Othman, R.; Saud, H.M. Effects of empty fruit bunch biochar and nitrogen-fixing bacteria on soil properties and growth of sweet corn. Malays. J. Soil Sci. 2016, 8, 177-194.

55. Tang, C.; Sparling, G.P.; McLay, C.D.A.; Raphael, C. Effect of short-term legume residue decomposition on soil acidity. Aust. J. Soil Res. 1999, 37, 561-573. [CrossRef]

56. Ch'ng, H.Y.; Ahmed, O.H.; Majid, N.M.N.A.; Jalloh, M.B. Improving soil phosphorus availability and yield of Zea mays L. using biochar and compost derived from agro-industrial wastes. Ital. J. Agron. 2019, 14, 34-42. [CrossRef]

57. Mensah, A.K.; Frimpong, K.A. Biochar and/or compost applications improve soil properties, growth, and yield of maize grown in acidic rainforest and coastal savannah soils in Ghana. Int. J. Agron. 2018, 2018. [CrossRef]

58. Panhwar, Q.A.; Naher, U.A.; Shamshuddin, J.; Ismail, M.R. Effects of biochar and ground magnesium limestone application, with or without bio-Fertilizer addition, on biochemical properties of an acid sulfate soil and rice yield. Agronomy 2020, 10, 1100. [CrossRef]

59. Masud, M.M.; Abdulaha-Al Baquyb, M.; Akhtera, S.; Sena, R.; Barmana, A.; Khatuna, M.R. Liming effects of poultry litter derived biochar on soil acidity amelioration and maize growth. Ecotoxicol. Environ. Saf. 2020, 202, 110865. [CrossRef]

60. Baquy, M.A.A.; Jiang, J.; Xu, R.K. Biochars derived from crop straws increased the availability of applied phosphorus fertilizer for maize in Ultisol and Oxisol. Environ. Sci. Pollut. Control Ser. 2020, 27, 5511-5522. [CrossRef] [PubMed]

61. Kamran, M.A.; Jiang, J.; Li, J.Y.; Shi, R.Y.; Mehmood, K.; Baquy, M.A.A.; Xu, R.K. Amelioration of soil acidity, Olsen-P, and phosphatase activity by manure- and peatderived biochars in different acidic soils. Arab. J. Geosci. 2018, 11, 272. [CrossRef]

62. Sohi, S.; Lopez-Capel, E.; Krull, E.S.; Bol, R. Biochar, climate change and soil: A review to guide future research. CSIRO Land Water Sci. Rep. 2009, 5, 1-57.

63. Steiner, C.; Teixeira, W.G.; Lehmann, J.; Nehls, T.; de Macêdo, J.L.V.; Blum, W.E.H.; Zech, W. Long-term effect of manure, charcoal and mineral fertilization on crop production and fertility on highly weathered central amazonian upland soil. Plant Soil 2007, 291, 275-290. [CrossRef]

64. Novak, J.M.; Busscher, W.J.; Laird, D.L.; Ahmedna, M.; Watts, D.W.; Niandou, M.A.S. Impact of biochar amendment on fertility of a southeastern Coastal Plain soil. Soil Sci. 2009, 174, 105-112. [CrossRef]

65. Mehmood, K.; Baquy, M.A.A.; Xu, R.K. Influence of nitrogen fertilizer forms and crop straw biochars on soil exchange properties and maize growth on an acidic Ultisol. Int. J. Agron. 2018, 64, 834-849. [CrossRef]

66. Biederman, L.A.; Harpole, W.S. Biochar and its effects on plant productivity and nutrient cycling: A meta-analysis. GCB Bioenergy 2013, 5, 202-214. [CrossRef]

67. Sheng, Y.; Zhan, Y.; Zhu, L. Reduced carbon sequestration potential of biochar in acidic soil. Sci. Total Environ. 2016, 572, 129-137. [CrossRef]

68. Rochette, P.; Angers, D.A.; Chantigny, M.H.; Gagnon, B.; Bertrand, N. In situ mineralization of dairy cattle manure as determined using soil-surface carbon dioxide fluxes. Soil Sci. Soc. Am. J. 2006, 70, 744-752. [CrossRef]

69. Mosharrof, M.; Uddin, M.K.; Sulaiman, M.F.; Mia, S.; Shamsuzzaman, S.M.; Haque, A.N.A. Combined Application of Biochar and Lime Increases Maize Yield and Accelerates Carbon Loss from an Acidic Soil. Agronomy 2021, 11, 1313. [CrossRef]

70. Fidel, R.B.; Laird, D.A.; Parkin, T.B. Effect of biochar on soil greenhouse gas emissions at the laboratory and field scales. Soil Syst. 2019, 3, 8. [CrossRef]

71. Chenfei, L.; Li, S.; Liang, C.; Xu, Q.; Li, Y.; Qin, H.; Fuhrmann, J.J. Response of microbial community structure and function to short-term biochar amendment in an intensively managed bamboo (Phyllostachys praecox) plantation soil: Effect of particle size and addition rate. Sci. Total Environ. 2017, 574, 24-33. [CrossRef] 
72. Mandal, S.; Sarkar, B.; Bolan, N.; Novak, J.; Ok, Y.S.; Van Zwieten, L.; Singh, B.P.; Kirkham, M.B.; Choppala, G.; Spokas, K.; et al. Designing advanced biochar products for maximizing greenhouse gas mitigation potential. Crit. Rev. Environ. Sci. Technol. 2016, 46, 1367-1401. [CrossRef]

73. Wang, Z.L.; Li, Y.F.; Chang, S.X.; Zhang, J.J.; Jiang, P.K.; Zhou, G.M.; Shen, Z.M. Contrasting effects of bamboo leaf and its biochar on soil CO2 efflux and labile organic carbon in an intensively managed Chinese chestnut plantation. Biol. Fertil. Soils 2014, 50, 1109-1119. [CrossRef] 